\title{
SAMPLING IN SPACES OF BANDLIMITED FUNCTIONS ON COMMUTATIVE SPACES
}

\author{
JENS GERLACH CHRISTENSEN AND GESTUR ÓLAFSSON
}

\begin{abstract}
A connected homogeneous space $\mathbf{X}=G / K$ is called commutative if $G$ is a connected Lie group, $K$ is a compact subgroup and the $B^{*}$-algebra $L^{1}(\mathbf{X})^{K}$ of $K$-invariant integrable function on $\mathbf{X}$ is commutative. In this article we introduce the space $L_{\Omega}^{2}(\mathbf{X})$ of $\Omega$-bandlimited function on $\mathbf{X}$ by using the spectral decomposition of $L^{2}(\mathbf{X})$. We show that those spaces are reproducing kernel Hilbert spaces and determine the reproducing kernel. We then prove sampling results for those spaces using the smoothness of the elements in $L_{\Omega}^{2}(\mathbf{X})$. At the end we discuss the example of $\mathbb{R}^{d}$, the spheres $S^{d}$, compact symmetric spaces and the Heisenberg group realized as the commutative space $\mathrm{U}(n) \ltimes \mathbb{H}_{n} / \mathrm{U}(n)$.
\end{abstract}

\section{INTRODUCTION}

Reconstruction or approximation of functions using the values of the function or a natural transformation of the function at discrete sets of points has an old and prominent history. A well known example is the reconstruction of a function using discrete set of line integrals, a fundamental tool in Computerized Tomography. Stepping into the digital age has only made this more important. But sampling theory as independent mathematical subject originates from the fundamental article [48]. We refer to [56], in particular the introduction, and [57] for a good places to consult about the history of the subject.

Sampling theory is a field of interest to engineers, signal analysts and mathematicians alike. It is concerned with the reconstruction of a function or signal from its values at a certain collection of points. Sampling theory is concerned with many questions:

(a) Which classes of signals can we expect to reconstruct?

(b) Which conditions do the sampling points have to satisfy?

(c) How are the signals reconstructed from samples?

(d) Reconstruction algorithms and error analysis.

(e) The speed of the reconstruction.

The first, and arguably most famous, result is the Whittaker-Shannon-Kotelnikov sampling theorem which states that an audible (band-limited) signal can be reconstructed from its values at equidistant sample points if the samples are taken at the Nyquist rate. If sampling takes place at a slower rate the signal cannot be reconstructed. With a higher sampling rate (oversampling) than the Nyquist rate the signal can be reconstructed by many different methods. Some of the developed methods also apply in the case when the samples are not equidistant (irregular sampling) but within the Nyquist rate.

Frames [12, 36 are generalization of bases and are relatively new addition to mathematics but have become increasingly important for approximation theory, reconstruction in function spaces, time frequency

2000 Mathematics Subject Classification. Primary 43A15,43A85; Secondary 46E22,22D12.

Key words and phrases. Sampling, bandlimited functions, reproducing kernel Hilbert spaces, Gelfand pairs, commutative spaces, representation theory, abstract harmonic analysis.

The research of J. G. Christensen was partially supported by NSF grant DMS-0801010, and ONR grants NAVY.N0001409103, NAVY.N000140910324.

The research of G. Ólafsson was supported by NSF Grant DMS-0801010. 
analysis analysis and generalizations to shift (translation) invariant spaces on topological groups and homogeneous spaces. So there is no surprise that frames have also been widely used in sampling theory. We will not go into detail here, but would like to point out the article by J. Benedetto 3 and by J. Benedetto and W. Heller [4] as well as the fundamental work by Feichtinger, Gröchenig and their coauthors [18, 19, 21, 24, 25, 26, 27, 32. Again, we refer to [56, in particular Chapter 10, for a good overview.

The natural generalization of the spaces of bandlimited functions on $\mathbb{R}^{n}$ are similarly defined translation invariant spaces of functions on Lie groups and homogeneous spaces, in particular homogeneous Riemannian spaces, a subject closely related to the coorbit theory of Feichtinger and Gröchenig and more general reproducing kernel Hilbert spaces related to unitary representations of Lie groups, [8, 9, 10, 20, 22, 23, 31. Here we have some natural tools from analysis at our disposal, including a natural algebra of invariant differential operators, in particular the Laplace operator. Mostly then the space of bandlimited functions are defined in terms of boundness of the eigenvalues of Laplace operator. The bounded geometry of the space allows us then to derive the needed Sobolev and Bernstein inequality. We point out 228, 29, 32, 33, 45, as important contributions to the subject.

This article is organized as follows. In Section 11 we recall some standard notation for Lie groups $G$ and homogeneous spaces $\mathbf{X}$. We introduce the algebra of invariant differential operators on homogeneous spaces and connect it with the algebra of invariant polynomials on $T_{x_{o}}(\mathbf{X})$, where $x_{o}$ is a fixed base-point. We then recall some basic fact about representations, and in particular, we introduce the space of smooth and analytic vectors. In 2 we discuss sampling in reproducing kernel Hilbert spaces on Lie groups. The main ideas are based on the work of Feichtinger and Gröchenig.

Section 3 deals with oscillation estimates on Lie groups. The exposition is based on [8] and uses smoothness of the the functions to derive oscillation results and hence sampling theorems.

We introduce the notion of Gelfand pairs and commutative spaces in Section 4. Here we assume that the group is connected, which allows us to state that $\mathbf{X}=G / K$ is commutative if and only if the algebra $\mathbb{D}(\mathbf{X})$ of $G$-invariant differential operators is commutative. We review well known facts about positive definite functions and the spherical Fourier transform on $\mathbf{X}$. One of the main results in this section is a recent theorem of Ruffino [46] which allows us to identify the parameter set for the spherical Fourier transform with a subset of $\mathbb{C}^{s}$, where $s$ is the number of generators for $\mathbb{D}(\mathbf{X})$. The result by Ruffino generalizes statements about Gelfand pairs related to the Heisenberg group by Benson, Jenkins, Ratcliff and Worku 7]. In Section [5 we relate the positive definite spherical functions to $K$-spherical representations of $G$ and introduce the vector valued Fourier transform on $\mathbf{X}$. This relates the representation theory of $G$ to the harmonic analysis on $\mathbf{X}$.

In Section 6 we finally introduce the space of bandlimited functions on $\mathbf{X}$. The definition is based on the support of the Plancherel-measure on $\mathbf{X}$ and not the spectrum of the Laplacian on $\mathbf{X}$ as in [28, 29, 45]. We do not prove it, but in all examples the definitions of a bandlimited functions are equivalent, but our definition allows for more general spectral sets. Another benefit of our approach is that one does not have to worry about injectivity radius for the exponential function nor about the construction of smooth partitions of unity (characteristic functions for a disjoint cover can be used just as well). Our sampling result is proved in 7 using a Bernstein inequality for the space of bandlimited functions. Finally, in Section 8 , we give some examples of commutative spaces and their spherical harmonic analysis. Those examples include the spheres, and more generally, compact symmetric spaces, and the Heisenberg group as a homogenous space for the group $\mathrm{U}(n) \ltimes \mathbb{H}_{n}$. This article is partially based on [1].

\section{Notation AND PRELIMINARIES}

1.1. Locally compact groups. In the following $G$ denotes a locally compact group with left invariant Haar measure $\mu_{G}$. Sometimes we write $d x$ instead of $d \mu_{G}(x)$. For $1 \leq p<\infty$ we let $L^{p}(G)$ denote the 
space of equivalence classes of $p$-integrable functions on $G$ with norm

$$
\|f\|_{L^{p}}=\left(\int|f(x)|^{p} d x\right)^{1 / p}
$$

Further, let $L^{\infty}(G)$ denote the space of essentially bounded function on $G$ with norm

$$
\|f\|_{\infty}=\operatorname{ess} \sup _{x \in G}|f(x)|
$$

The spaces $L^{p}(G)$ are Banach spaces for $1 \leq p \leq \infty$ and $L^{2}(G)$ is a Hilbert space with inner product

$$
(f, g)=\int f(x) \overline{g(x)} d x
$$

When it makes sense (either the integrand is integrable or as a vector valued integral) we define the convolution

$$
f * g(x)=\int f(y) g\left(y^{-1} x\right) d y
$$

Equipped with convolution the space $L^{1}(G)$ becomes a Banach algebra. For functions on $G$ we denote the left and right translations by

$$
\ell(a) f(x)=f\left(a^{-1} x\right) \quad \text { and } \quad \rho(a) f(x)=f(x a)
$$

respectively. Now, let $K$ be a compact subgroup of $G$ with bi-invariant Haar measure $\mu_{K}$. We always normalize $\mu_{K}$ so that $\mu_{K}(K)=1$. The same convention applies to other compact groups and compact spaces.

If $\mathcal{A}$ is a set of functions on $G$ we denote the left $K$-fixed subset as

$$
\mathcal{A}^{K}=\{f \in \mathcal{A} \mid \ell(k) f=f\}
$$

and similarly the right $K$-fixed subset is denoted

$$
\mathcal{A}^{\rho(K)}=\{f \in \mathcal{A} \mid \rho(k) f=f\}
$$

Let $\mathbf{X}=G / K, x_{0}=e K$ and let $\kappa: G \rightarrow \mathbf{X}$ be the canonical map $g \mapsto g x_{0}$. We will identify functions on $\mathbf{X}$ by $K$-invariant functions via $f \leftrightarrow f \circ \kappa$. The space $\mathbf{X}$ possesses a $G$-invariant measure $\mu_{\mathbf{X}}$ and, since $K$ is compact, the $L^{p}$-spaces

$$
L^{p}(\mathbf{X})=\left\{f \mid f \text { is } \mu_{\mathbf{X}} \text {-measurable and } \int|f(x)|^{p} d \mu_{\mathbf{X}}<\infty\right\} .
$$

The above map $f \mapsto f \circ \kappa$ is an isometric isomorphism $L^{p}(\mathbf{X}) \simeq L^{p}(G)^{\rho(G)}$. In particular, $L^{p}(\mathbf{X})$ is a closed $G$-invariant subspace of $L^{p}(G)$. The projection $L^{p}(G) \rightarrow L^{p}(\mathbf{X})$ is

$$
p_{K}(f)(x)=\int_{K} f(x k) d \mu_{K}(k) .
$$

If $f \in L^{1}(G)$ and $g \in L^{p}(\mathbf{X}), 1 \leq p \leq \infty$, then $f * g \in L^{p}(\mathbf{X})$ and $\|f * g\|_{p} \leq\|f\|_{1}\|g\|_{p}$.

If $f$ is further assumed to be left $K$-invariant, then

$$
\begin{aligned}
f * g(k y) & =\int_{G} f(x) g\left(x^{-1} k y\right) d \mu_{G}(x) \\
& =\int_{G} f(k x) g\left(x^{-1} y\right) d \mu_{G}(x) \\
& =\int_{G} f(x) g\left(x^{-1} y\right) d \mu_{G}(x) \\
& =f * g(y) .
\end{aligned}
$$


Thus $f * g$ is also left $K$-invariant. Denote by $m_{G}$ the modular function on $G$. Note that $m_{G}$ is usually denoted by $\Delta$ or $\Delta_{G}$ but we will need that notation for the Laplace operator on $\mathbf{X}$ respectively $G$. We have $\left.m_{G}\right|_{K}=1$ as $K$ is compact. Hence $m_{G}$ is $K$-biinvariant. It follows that $L^{1}(\mathbf{X})^{K}$ is invariant under the anti-involutions $f^{\vee}(x)=m_{G}(x)^{-1} f\left(x^{-1}\right)$ and $f^{*}=\overline{f^{\vee}}$. In particular, $L^{1}(\mathbf{X})^{K}$ is a closed Banach *-subalgebra of $L^{1}(G)$.

1.2. Lie theory. Let $G$ be a connected Lie group and $K$ a compact subgroup. Most of the statements holds for nonconnected groups, but some technical problems turn up as we start to deal with the Lie algebra and invariant differential operators. We will therefore for simplicity assume $G$ commutative from the beginning.

Denote by $\mathfrak{g}$ the Lie algebra of $G$ and $\mathfrak{k}$ the Lie algebra of $K$. Fix a $K$-invariant inner product $\langle$,$\rangle on$ $\mathfrak{g}$. That is always possible as $K$ is compact. Let $\mathfrak{s}:=\mathfrak{k}^{\perp}$. Then $\mathfrak{s}$ is $K$-invariant and $\mathfrak{s} \simeq T_{x_{o}}(\mathbf{X})($ as a $K$-module) via the map

$$
X \mapsto D(X), \quad D(X)(f)\left(x_{o}\right):=\left.\frac{d}{d t}\right|_{t=0} f\left(\exp (t X) x_{o}\right) .
$$

Denote also by $\langle$,$\rangle the restriction of \langle$,$\rangle to \mathfrak{s} \times \mathfrak{s}$. As the tangent bundle on $T(\mathbf{X})$ is isomorphic to $G \times{ }_{K} \mathfrak{s}$ as a $G$-bundle it follows that the restriction of $\langle$,$\rangle to \mathfrak{s}$ defines a $G$-invariant Riemannian structure on $\mathbf{X}$.

Let $D: C_{c}^{\infty}(\mathbf{X}) \rightarrow C_{c}^{\infty}(\mathbf{X})$ be a differential operator. For $g \in G$ let $g \cdot D: C_{c}^{\infty}(\mathbf{X}) \rightarrow C_{c}^{\infty}(\mathbf{X})$ be the differential operator

$$
g \cdot D(f)(x)=D\left(\ell\left(g^{-1}\right) f\right)\left(g^{-1} x\right) .
$$

$D$ is said to be $G$-invariant if $g \cdot D=D$ for all $g \in G$. Thus $D$ is $G$-invariant if and only if $D$ commutes with left translation, $D(\ell(g) f)=\ell(g) D(f)$. Denote by $\mathbb{D}(\mathbf{X})$ the algebra of $G$-invariant differential operators on $\mathbf{X}$. The algebra $\mathbb{D}(\mathbf{X})$ has a simple description. For a polynomial function $p: \mathfrak{g} \rightarrow \mathbb{C}$ define a left-invariant differential operator $D_{p}: C_{c}^{\infty}(G) \rightarrow C_{c}^{\infty}(G)$ by

$$
\begin{aligned}
D_{p}(f)(g) & :=\left.p\left(\frac{\partial}{\partial t_{1}}, \ldots, \frac{\partial}{\partial t_{m}}\right) f\left(g \exp \left(t_{1} X_{1}+\ldots+t_{m} X_{m}\right)\right)\right|_{t_{1}=\ldots=t=0} \\
& =\left.p\left(\frac{\partial}{\partial t_{1}}, \ldots, \frac{\partial}{\partial t_{m}}\right) f\left(g \exp \left(t_{1} X_{1}\right) \cdots \exp \left(t_{m} X_{m}\right)\right)\right|_{t_{1}=\ldots=t=0}
\end{aligned}
$$

where we have extended our basis of $\mathfrak{s}$ to a basis $X_{1}, \ldots, X_{m}$ of $\mathfrak{g}$. If $p$ is a $K$-invariant then

$$
\begin{aligned}
D_{p}(f)(g k) & =\left.p\left(\frac{\partial}{\partial t_{1}}, \ldots, \frac{\partial}{\partial t_{m}}\right) f\left(g k \exp \left(t_{1} X_{1}+\ldots+t_{n} X_{m}\right)\right)\right|_{t_{1}=\ldots=t_{m}=0} \\
& =\left.p\left(\frac{\partial}{\partial t_{1}}, \ldots, \frac{\partial}{\partial t_{n}}\right) f\left(g \exp \left(t_{1} X_{1}+\ldots+t_{m} X_{m}\right) k\right)\right|_{t_{1}=\ldots=t_{m}=0}
\end{aligned}
$$

for all $k \in K$. Hence, if $p$ is $K$-invariant and $f$ is right $K$-invariant it is clear from (2) that $D_{p}$ only depends on the polynomial $q=\left.p\right|_{\mathfrak{s}}$ and $D_{p} f=D_{q} f$ is right $K$-invariant and defines a function on $\mathbf{X}$. Hence $D_{q}$ is a $G$-invariant differential operator on $\mathbf{X}$.

Denote by $S(\mathfrak{s})$ the symmetric algebra over $\mathfrak{s}$. Then $S(\mathfrak{s})$ is commutative and isomorphic to the algebra of polynomial functions.

Theorem 1.1. The map $S(\mathfrak{s})^{K} \rightarrow \mathbb{D}(\mathbf{X})$ is bijective.

Proof. This is Theorem 10 in [37.

Remark 1.2. If we take $p(X)=\|X\|^{2}$, then $D_{p}=: \Delta$ is the Laplace operator on $\mathbf{X}$.

Remark 1.3. The algebra $\mathbb{D}(\mathbf{X})$ is not commutative in general. Hence the above map is not necessarily an algebra homomorphism. 
For a fixed basis $X_{1}, \ldots, X_{m}$ for $\mathfrak{g}$ it will ease our notation to introduce the differential operator $D^{\alpha}$ : $C_{c}^{\infty}(G) \rightarrow C_{c}^{\infty}(G)$ for a multi-index $\alpha$ of length $k$ with entries between 1 and $m$ :

$$
D^{\alpha} f(x)=D\left(X_{\alpha(k)}\right) D\left(X_{\alpha(k-1)}\right) \cdots D\left(X_{\alpha(1)}\right) f(x) .
$$

1.3. Representation theory. Let $\pi$ be a representation of the Lie group $G$ on a Hilbert space $\mathcal{H}$. Then $u \in \mathcal{H}$ is called smooth respectively analytic if the $\mathcal{H}$-valued function $\pi_{u}(x)=\pi(x) u$ is smooth respectively analytic. Denote by $\mathcal{H}^{\infty}$, respectively $\mathcal{H}^{\omega}$, the space of smooth, respectively analytic, vectors in $\mathcal{H}$. For $u \in \mathcal{H}^{\infty}$ and $X \in \mathfrak{g}$ let

$$
\pi^{\infty}(X) u:=\lim _{t \rightarrow 0} \frac{\pi(\exp t X) u-u}{t}
$$

and $\pi^{\omega}(X):=\left.\pi^{\infty}(X)\right|_{\mathcal{H}^{\omega}}$. We have

Lemma 1.4. Let $(\pi, \mathcal{H})$ be a unitary representation of $G$. Then the following holds:

(a) The space $\mathcal{H}^{\infty}$ is $G$-invariant.

(b) $\pi^{\infty}(\mathfrak{g}) \mathcal{H}^{\infty} \subseteq \mathcal{H}^{\infty}$ and $\left(\pi^{\infty}, \mathcal{H}^{\infty}\right)$ is a representation of $\mathfrak{g}$. In particular

$$
\pi^{\infty}([X, Y])=\pi^{\infty}(X) \pi^{\infty}(Y)-\pi^{\infty}(Y) \pi^{\infty}(X)
$$

(c) $\pi^{\infty}(\operatorname{Ad}(g) X)=\pi(g) \pi^{\infty}(X) \pi\left(g^{-1}\right)$,

(d) $\left.\pi^{\infty}(X)^{*}\right|_{\mathcal{H}}=-\pi^{\infty}(X)$.

(e) $\mathcal{H}^{\infty}$ is dense in $\mathcal{H}$.

Corresponding statements are also true for $\mathcal{H}^{\omega}$. To show that $\mathcal{H}^{\infty}$ is dense in $\mathcal{H}$ let $f \in L^{1}(G)$. Define $\pi(f): \mathcal{H} \rightarrow \mathcal{H}$ by

$$
\pi(f) u=\int_{G} f(x) \pi(x) u d \mu_{G}(x) .
$$

Then $\|\pi(f)\| \leq\|f\|_{1}, \pi(f * g)=\pi(f) \pi(g)$ and $\pi\left(f^{*}\right)=\pi(f)^{*}$. Thus, $\pi: L^{1}(G) \rightarrow B(\mathcal{H})$ is a continuous *-homomorphism. If $f \in C_{c}^{\infty}(G)$ then it is easy to see that $\pi(f) \mathcal{H} \subseteq \mathcal{H}^{\infty}$. The main step in the proof is to show that

$$
\pi^{\infty}(X) \pi(f) u=\pi\left(\ell^{\infty}(X) f\right) u
$$

where

$$
\ell^{\infty}(X) f(x)=\lim _{t \rightarrow 0} \frac{f(\exp (-t X) x)-f(x)}{t} .
$$

Lemma 1.5. If $\left\{U_{j}\right\}$ is a decreasing sequence of e-neighborhoods such that $\bigcap U_{j}=\{e\}$ and $f_{j} \in C_{c}^{\infty}(G)$ is so that $f_{j} \geq 0, \operatorname{supp} f_{j} \subset U_{j}$, and $\|f\|_{1}=1$, then $\pi\left(f_{j}\right) u \rightarrow u$ for all $u \in \mathcal{H}$. In particular, $\mathcal{H}^{\infty}$ is dense in $\mathcal{H}$.

\section{Reconstruction in Reproducing kernel Hilbert spaces}

We will be concerned with sampling in subspaces of the Hilbert space $L^{2}(G)$. We start with the definition of a frame due to Duffin and Schaeffer [15]. For further references see the introduction.

Definition 2.1. For a Hilbert space $\mathcal{H}$ a set of vectors $\left\{\phi_{i}\right\} \subseteq \mathcal{H}$ is a frame for $\mathcal{H}$ if there are constants $0<A \leq B<\infty$ such that

$$
A\|f\|_{\mathcal{H}}^{2} \leq \sum_{i}\left|\left(f, \phi_{i}\right)\right|^{2} \leq B\|f\|_{H}^{2}
$$

for all $f \in \mathcal{H}$. 
These conditions ensure that the frame operator $S: \mathcal{H} \rightarrow \mathcal{H}$ given by

$$
S f=\sum_{i}\left(f, \phi_{i}\right) \phi_{i}
$$

is invertible and that $f$ can be reconstructed by

$$
f=\sum_{i}\left(f, \phi_{i}\right) \psi_{i}
$$

where $\psi_{i}=S^{-1} \phi_{i}$. The sequence $\left\{\psi_{i}\right\}$ is also a frame called the dual frame. In general there are other ways to reconstruct $f$ form the sequence $\left\{\left(f, \phi_{i}\right)\right\}$.

The inversion of $S$ can be carried out via the Neumann series

$$
S^{-1}=\frac{2}{A+B} \sum_{n=0}^{\infty}\left(I-\frac{2}{A+B} S\right)^{n}
$$

which has rate of convergence $\left\|I-\frac{2}{A+B} S\right\| \leq \frac{B-A}{A+B}$ (which is the best possible for optimal frame bounds [42, 34, 43]).

One way to reconstruct $f$ from samples is to assume that the point evaluations $f \mapsto f(x)$ is continuous and hence given by an inner product $f(x)=\left(f, g_{x}\right), g_{x} \in \mathcal{H}$, and that there exists a sequence $\left\{x_{j}\right\}$ in $G$ such that $\left\{g_{x_{j}}\right\}$ is a frame. A reasonable class of functions to work with are therefore reproducing kernel Hilbert spaces. A classical reference for reproducing kernel Hilbert spaces is [1]. A Hilbert space $\mathcal{H}$ of functions on $G$ is called a reproducing kernel Hilbert space if point evaluation is continuous, i.e. if for every $x \in G$ there is a constant $C_{x}$ such that for all $f \in \mathcal{H}$

$$
|f(x)| \leq C_{x}\|f\|_{\mathcal{H}}
$$

Here are the main facts about closed reproducing kernel subspaces of $L^{2}(G)$ which is all what we will need here:

Proposition 2.2. If $\mathcal{H}$ is a closed and left invariant reproducing kernel subspace of $L^{2}(G)$ then

(a) There is a $\phi \in \mathcal{H}$ such that $f=f * \phi$ for all $f \in \mathcal{H}$.

(b) The functions in $\mathcal{H}$ are continuous.

(c) The kernel $\phi$ satisfies $\overline{\phi\left(x^{-1}\right)}=\phi(x)$ so $f(x)=f * \phi(x)=(f, \ell(x) \phi)$.

(d) The mapping $f \mapsto f * \phi$ is a continuous projection from $L^{2}(G)$ onto $\mathcal{H}$. In particular $\mathcal{H}=\{f \in$ $\left.L^{2}(G) \mid f * \phi=f\right\}$.

Proof. Here are the main ideas of the proof. By Riesz' representation theorem there is a $g_{x} \in H$ such that

$$
f(x)=\int f(y) \overline{g_{x}(y)} d y .
$$

Let $g(x):=g_{e}(x)$. The left invariance of $\mathcal{H}$ ensures that

$$
f(x)=\left[\ell\left(x^{-1}\right) f\right](e)=\int f(x y) \overline{g_{e}(y)} d y=\int f(y) \overline{g\left(x^{-1} y\right)} d y=(f, \ell(x) g) .
$$

Hence $g_{x}(y)=g\left(x^{-1} y\right)$. We also have

$$
g\left(x^{-1} y\right)=\left(g_{x}, g_{y}\right)=\overline{\left(g_{y}, g_{x}\right)}=\overline{g\left(y^{-1} x\right)} .
$$

Thus, if we set $\phi(x)=\overline{g\left(x^{-1}\right)}$, which agrees with $g^{*}$ in case $G$ is unimodular, we get $f=f * \phi$, which in particular implies that $\mathcal{H} \subseteq C(G)$ as claimed.

Assume that $f * \phi=f$ and that $f \perp \mathcal{H}$. Then $f(x)=\left(f, g_{x}\right)=0$ as $g_{x} \in \mathcal{H}$. Hence $f=0$ and $\mathcal{H}=L^{2}(G) * \phi=\left\{f \in L^{2}(G) \mid f * \phi=f\right\}$. 
Remark 2.3. It should be noted that several functions $\phi \in L^{2}(G)$ could satisfy $f=f * \phi$ for $f \in \mathcal{H}$. Just take an arbitrary function $\eta$ such that $\overline{\eta^{\vee}} \in \mathcal{H}^{\perp}$. Then $f *(\phi+\eta)=f * \phi$. The restriction that $\phi \in \mathcal{H}$ ensures uniqueness of $\phi$. Example could be sinc functions for spaces of larger bandwidth than $\mathcal{H}$.

The sampling theory of Feichtinger, Gröchenig and Führ, see the introduction for referecnes, builds on estimation of the variation of a function under small right translations. The local oscillations were therefore introduced as follows: For a compact neighbourhood $U$ of the identity define

$$
\operatorname{osc}_{U}(f)=\sup _{u \in U}\left|f(x)-f\left(x u^{-1}\right)\right|
$$

Before stating the next result we need to introduce a reasonable collection of points at which to sample: For a compact neighbourhood $U$ of the identity, the points $x_{i}$ are called $U$-relatively separated if the $x_{i} U$ cover $G$ and there is an $N$ such that each $x \in G$ belongs to at most $N$ of the $x_{i} U$ 's.

Lemma 2.4. Let $\mathcal{H}$ be a reproducing kernel subspace $L^{2}(G)$ with reproducing convolution kernel $\phi$. Assume that for any compact neighbourhood $U$ of the identity there is a constant $C_{U}$ such that for any $f \in \mathcal{H}$ the estimate $\left\|\operatorname{osc}_{U}(f)\right\|_{L^{2}} \leq C_{U}\|f\|_{\mathcal{H}}$ holds. If we can choose $U$ such that $C_{U}<1$, then for any $U$-relatively separated points $\left\{x_{i}\right\}$ the norms $\left\|\left\{f\left(x_{i}\right)\right\}\right\|_{\ell^{2}}$ and $\|f\|_{L^{2}}$ are equivalent, and $\ell\left(x_{i}\right) \phi$ forms a frame for $\mathcal{H}$.

Proof.

$$
\begin{aligned}
\left\|\left\{f\left(x_{i}\right)\right\}\right\|_{\ell^{2}}^{2} & =|U|^{-1}\left\|\sum_{i}\left|f\left(x_{i}\right)\right|^{2} \mathbf{1}_{x_{i} U}\right\|_{L^{1}} \\
& \leq|U|^{-1}\left\|\sum_{i}\left|f\left(x_{i}\right)\right| \mathbf{1}_{x_{i} U}\right\|_{L^{2}}^{2} \\
& \leq|U|^{-1}\left(\left\|\sum_{i}\left|f\left(x_{i}\right)-f\right| \mathbf{1}_{x_{i} U}\right\|_{L^{2}}+\left\|\sum_{i}|f| \mathbf{1}_{x_{i} U}\right\|_{L^{2}}\right)^{2} \\
& \leq|U|^{-1}\left(\left\|\sum_{i}\left|\operatorname{osc}_{U}(f)\right| \mathbf{1}_{x_{i} U}\right\|_{L^{2}}+\left\|\sum_{i}|f| \mathbf{1}_{x_{i} U}\right\|_{L^{2}}\right)^{2} \\
& \leq|U|^{-1} N^{2}\left(\left\|\operatorname{osc}_{U}(f)\right\|_{L^{2}}+\|f\|_{L^{2}}\right)^{2} \\
& \leq|U|^{-1} N^{2}\left(1+C_{U}\right)^{2}\|f\|_{L^{2}}^{2} .
\end{aligned}
$$

Here $N$ is the maximal number of overlaps between the $x_{i} U$ 's. To get the other inequality we let $\psi_{i}$ be a bounded partition of unity such that $0 \leq \psi_{i} \leq \mathbf{1}_{x_{i}} U$ and $\sum_{i} \psi_{i}=1$. Then,

$$
\begin{aligned}
\|f\|_{L^{2}} & \leq\left\|f-\sum_{i} f\left(x_{i}\right) \psi_{i}\right\|_{L^{2}}+\left\|\sum_{i} f\left(x_{i}\right) \psi_{i}\right\|_{L^{2}} \\
& \leq\left\|\sum_{i} \operatorname{osc}_{U}(f) \psi_{i}\right\|_{L^{2}}+\left\|\sum_{i}\left|f\left(x_{i}\right)\right| \mathbf{1}_{x_{i} U}\right\|_{L^{2}} \\
& \leq\left\|\operatorname{osc}_{U}(f)\right\|_{L^{2}}+N|U|\left\|f\left(x_{i}\right)\right\|_{\ell^{2}} \\
& \leq C_{U}\|f\|_{L^{2}}+N|U|\left\|f\left(x_{i}\right)\right\|_{\ell^{2}}
\end{aligned}
$$

If $C_{U}<1$ then we get

$$
\left(1-C_{U}\right)\|f\|_{L^{2}} \leq N|U|\left\|f\left(x_{i}\right)\right\|_{\ell^{2}} .
$$

This concludes the proof.

Remark 2.5. From the proof of the lemma follows that the the norm equivalence becomes

$$
\left(\frac{1-C_{U}}{|U| N}\right)^{2}\|f\|_{L^{2}}^{2} \leq\left\|\left\{f\left(x_{i}\right)\right\}\right\|_{\ell^{2}}^{2} \leq\left(N \frac{1+C_{U}}{|U|}\right)^{2}\|f\|_{L^{2}}^{2},
$$


and thus the frame constants $A$ and $B$ can be chosen to be

$$
A=\left(\frac{1-C_{U}}{|U| N}\right)^{2} \quad \text { and } \quad B=\left(N \frac{1+C_{U}}{|U|}\right)^{2} .
$$

It follows that the rate of convergence for the Neumann series (3) can be estimated by

$$
\frac{B-A}{B+A}=\frac{N^{2}\left(1+C_{U}\right)^{2}-\left(1-C_{U}\right)^{2} / N^{2}}{N^{2}\left(1+C_{U}\right)^{2}+\left(1-C_{U}\right)^{2} / N^{2}} \rightarrow \frac{N^{4}-1}{N^{4}+1} \quad \text { as } C_{U} \rightarrow 0 .
$$

This shows that as the sampling points $x_{i}$ are chosen closer ( $U$ gets smaller) the rate of convergence can be very slow (assuming that we can choose the overlaps of the $x_{i} U$ 's bounded by a certain $N$ even if $U$ gets smaller). We therefore have very little control of the rate of convergence in this case.

To obtain operators with faster decaying Neumann series, Feichtinger and Gröchenig introduced new sampling operators. An example is the sampling operator $T: \mathcal{H} \rightarrow \mathcal{H}$ defined as

$$
T f=\sum_{i} f\left(x_{i}\right) \psi_{i} * \phi .
$$

Using oscillations it is possible to estimate the norm of $I-T$ by $C_{U}$ :

$$
\|f-T f\|_{L^{2}}=\left\|\left(\sum_{i}\left|f-f\left(x_{i}\right)\right| \psi_{i}\right) * \phi\right\|_{L^{2}} \leq\left\|\operatorname{osc}_{U} f\right\|_{L^{2}} \leq C_{U}\|f\|_{L^{2}}
$$

Thus $T$ is invertible on $L_{\phi}^{2}$ if $C_{U}<1$ with rate of convergence of Neumann series governed directly by $C_{U}$. By increasing the rate of sampling (decreasing $U$ and thereby $C_{U}$ ) fewer iterations are necessary in order to obtain good approximation. This was not the case for the frame inversion above.

\section{Oscillation estimates on Lie Groups}

In this section we will show how oscillation estimates can be obtained for functions on Lie groups.

First we set up the notation. As before we let $G$ be a Lie group with Lie algebra $\mathfrak{g}$. Fix a basis $\left\{X_{i}\right\}_{i=1}^{\operatorname{dim}(G)}$ for $\mathfrak{g}$. Denote by $U_{\epsilon}$ the set

$$
U_{\epsilon}:=\left\{\exp \left(t_{1} X_{1}\right) \cdots \exp \left(t_{n} X_{n}\right) \mid-\epsilon \leq t_{k} \leq \epsilon, 1 \leq k \leq n\right\} .
$$

Remark 3.1. Note that $U_{\epsilon}$ depends on the choice of basis as well as the ordering of the vectors. It would therefore be more natural to use sets of the form $V_{\epsilon}:=\exp \{X \in \mathfrak{g} \mid\|X\| \leq \epsilon\}$ or even $W_{\epsilon}:=\exp \{X \in$ $\mathfrak{s} \mid\|X\| \leq \epsilon\} \exp \{X \in \mathfrak{k} \mid\|X\| \leq \epsilon\}$. Both of those sets are invariant under conjugation by elements in $K$. The reason to use $U_{\epsilon}$ as defined above is, that this is the definition that works best for the proofs! But it should be noted that $V_{\epsilon}, W_{\epsilon} \subseteq U_{\epsilon}$. Hence the local oscillation using either $V_{\epsilon}$ or $W_{\epsilon}$ is controlled by the local oscillation using $U_{\epsilon}$.

Set

$$
\operatorname{osc}_{\epsilon}(f)=\operatorname{osc}_{U_{\epsilon}}(f) .
$$

By $\delta$ we denote an $n$-tuple $\delta=\left(\delta_{1}, \ldots, \delta_{n}\right)$ with $\delta_{i} \in\{0,1\}$. The length $|\delta|$ of $\delta$ is the number of non-zero entries $|\delta|=\delta_{1}+\cdots+\delta_{n}$. Further, define the function $\tau_{\delta}:(-\epsilon, \epsilon)^{n} \rightarrow G$ by

$$
\tau_{\delta}\left(t_{1}, \ldots, t_{n}\right)=\exp \left(\delta_{1} t_{n} X_{1}\right) \cdots \exp \left(\delta_{n} t_{1} X_{n}\right) .
$$

Lemma 3.2. If $f$ is right differentiable of order $n=\operatorname{dim}(G)$ then there is a constant $C_{\epsilon}$ such that

$$
\operatorname{osc}_{\epsilon}(f)(x) \leq C_{\epsilon} \sum_{1 \leq|\alpha| \leq n|\delta|=|\alpha|} \sum_{|\delta| \text { integrals }}^{\int_{-\epsilon}^{\epsilon} \cdots \int_{-\epsilon}^{\epsilon}}\left|D^{\alpha} f\left(x \tau_{\delta}\left(t_{1}, \ldots, t_{n}\right)^{-1}\right)\right|\left(d t_{1}\right)^{\delta_{1}} \cdots\left(d t_{n}\right)^{\delta_{n}} .
$$


For $\epsilon^{\prime} \leq \epsilon$ we have $C_{\epsilon^{\prime}} \leq C_{\epsilon}$.

Proof. We refer to $[8$ for a full proof. Instead we restrict ourselves to a proof in 2 dimension that easily carries over to arbitrary dimensions. We will sometimes write $e^{X}$ instead of $\exp X$.

For $y \in U_{\epsilon}$ there are $s_{1}, s_{2} \in[-\epsilon, \epsilon]$ such that $y^{-1}=e^{-s_{2} X_{2}} e^{-s_{1} X_{1}}$. Hence

$$
\begin{aligned}
\left|f(x)-f\left(x y^{-1}\right)\right| & =\left|f(x)-f\left(x e^{-s_{2} X_{2}} e^{-s_{1} X_{1}}\right)\right| \\
& \leq\left|f(x)-f\left(x e^{-s_{2} X_{2}}\right)\right|+\left|f\left(x e^{-s_{2} X_{2}}\right)-f\left(x e^{-s_{2} X_{2}} e^{-s_{1} X_{1}}\right)\right| \\
& =\left|\int_{0}^{s_{2}} \frac{d}{d t_{2}} f\left(x e^{-t_{2} X_{2}}\right) d t_{2}\right|+\left|\int_{0}^{s_{1}} \frac{d}{d t_{1}} f\left(x e^{-s_{2} X_{2}} e^{-t_{1} X_{1}}\right) d t_{1}\right| \\
& \leq \int_{-\epsilon}^{\epsilon}\left|D\left(X_{2}\right) f\left(x e^{-t_{2} X_{2}}\right)\right| d t_{2}+\int_{-\epsilon}^{\epsilon}\left|D\left(X_{1}\right) f\left(x e^{-s_{2} X_{2}} e^{-t_{1} X_{1}}\right)\right| d t_{1} .
\end{aligned}
$$

Since

$$
e^{-s_{2} X_{2}} e^{-t_{1} X_{1}}=e^{-t_{1} X_{1}} e^{-s_{2} X\left(t_{1}\right)} \quad \text { with } \quad X(t)=\operatorname{Ad}\left(\exp \left(t X_{1}\right)\right) X_{2}
$$

the term $\left|D\left(X_{1}\right) f\left(x e^{-s_{2} X_{2}} e^{-t_{1} X_{1}}\right)\right|$ can be estimated by

$$
\begin{aligned}
\mid D\left(X_{1}\right) & f\left(x e^{-s_{2} X_{2}} e^{-t_{1} X_{1}}\right) \mid \\
= & \left|D\left(X_{1}\right) f\left(x e^{-t_{1} X_{1}} e^{-s_{2} X\left(t_{1}\right)}\right)\right| \\
\leq & \left|D\left(X_{1}\right) f\left(x e^{-t_{1} X_{1}} e^{-s_{2} X\left(t_{1}\right)}\right)-D\left(X_{1}\right) f\left(x e^{-t_{1} X_{1}}\right)\right|+\left|D\left(X_{1}\right) f\left(x e^{-t_{1} X_{1}}\right)\right| \\
= & \left|\int_{0}^{s_{1}} \frac{d}{d t_{2}} D\left(X_{1}\right) f\left(x e^{-t_{1} X_{1}} e^{-t_{2} X\left(t_{1}\right)}\right) d t_{2}\right|+\left|D\left(X_{1}\right) f\left(x e^{-t_{1} X_{1}}\right)\right| \\
= & \left|\int_{0}^{s_{1}} D\left(X\left(t_{1}\right)\right) D\left(X_{1}\right) f\left(x e^{-t_{1} X_{1}} e^{-t_{2} X\left(t_{1}\right)}\right) d t_{2}\right|+\left|X_{1} f\left(x e^{-t_{1} X_{1}}\right)\right| \\
\leq & C_{\epsilon} \int_{-\epsilon}^{\epsilon}\left|D\left(X_{2}\right) D\left(X_{1}\right) f\left(x e^{-t_{2} X_{2}} e^{-t_{1} X_{1}}\right)\right|+\left|D\left(X_{1}\right) D\left(X_{1}\right) f\left(x e^{-t_{2} X_{2}} e^{-t_{1} X_{1}}\right)\right| d t_{2} \\
& \quad+\left|D\left(X_{1}\right) f\left(x e^{-t_{1} X_{1}}\right)\right| .
\end{aligned}
$$

The last inequality follows since $D\left(X\left(t_{2}\right)\right)=a\left(t_{1}\right) D\left(X_{1}\right)+b\left(t_{1}\right) D\left(X_{2}\right)$ is a differential operator with coefficients $a$ and $b$ depending continuously, in fact analytically, on all variables. Together (5) and (6) provide the desired estimate.

Since right translation is continuous on $L^{2}(G)$ and $\sup _{u \in U}\left\|r_{u} f\right\|_{L^{2}} \leq C_{U}\|f\|_{L^{2}}$ for compact $U$ [55, Theorem 3.29] gives

$$
\begin{aligned}
\left\|\operatorname{osc}_{\epsilon}(f)\right\|_{L^{2}} & \leq \sum_{1 \leq|\alpha| \leq n|\delta|=|\alpha|} \sum_{|\delta| \text { integrals }} \underbrace{\epsilon}_{-\epsilon} \cdots \int_{-\epsilon}^{\epsilon}\left\|r_{\tau_{\delta}\left(t_{1}, \ldots, t_{n}\right)^{-1}} D^{\alpha} f\right\|_{L^{2}}\left(d t_{1}\right)^{\delta_{1}} \cdots\left(d t_{n}\right)^{\delta_{n}} \\
& \leq C_{U_{\epsilon}} \sum_{1 \leq|\alpha| \leq n} \sum_{|\delta|=|\alpha|} \underbrace{\int_{-\epsilon}^{\epsilon} \cdots \int_{-\epsilon}^{\epsilon}\left\|D^{\alpha} f\right\|_{L^{2}}\left(d t_{1}\right)^{\delta_{1}} \cdots\left(d t_{n}\right)^{\delta_{n}}}_{|\delta| \text { integrals }} \\
& \leq C_{U_{\epsilon}} \sum_{1 \leq|\alpha| \leq n}\left(\begin{array}{c}
n \\
|\alpha|
\end{array}\right) \epsilon^{|\alpha|}\left\|D^{\alpha} f\right\|_{L^{2}}
\end{aligned}
$$

To sum up we get 
Theorem 3.3. If $D^{\alpha} f \in L^{2}(G)$ for all $|\alpha| \leq n$, then

$$
\left\|\operatorname{osc}_{\epsilon}(f)\right\|_{L^{2}} \leq C_{\epsilon} \sum_{1 \leq|\alpha| \leq n}\left\|D^{\alpha} f\right\|_{L^{2}}
$$

where $C_{\epsilon} \rightarrow 0$ as $\epsilon \rightarrow 0$.

We will need the following fact later when we obtain a Bernstein type inequality for band-limited functions on a commutative space. If $\langle X, Y\rangle$ defines an inner product on $\mathfrak{g}$ and $X_{1}, \ldots, X_{n}$ is an orthonormal basis, then the associated Laplace operator has the form $\Delta_{G}=D\left(X_{1}\right)^{2}+\cdots+D\left(X_{n}\right)^{2}$. We have:

Lemma 3.4. Let the notation be as above. Then

$$
\sum_{1 \leq|\alpha| \leq n}\left\|D^{\alpha} f\right\|_{L^{2}} \leq C\left\|\left(I-\Delta_{G}\right)^{n / 2} f\right\|_{L^{2}}
$$

Proof. According to Theorem 4 in [52] the Sobolev norm on the left can be estimated by the Bessel norm, defined in 49 , on the right.

\section{Gelfand Pairs and Commutative Spaces}

In this section we introduce the basic notation for Gelfand pairs and commutative spaces. Our standard references are [13, Chapter 22, 14, 39, Chapter IV, and [54. We give several examples in Section 8 .

Let $G$ be a connected Lie group and $K$ a compact subgroup.

Theorem 4.1. Suppose that $G$ is a connected Lie group and $K$ a compact subgroup. Then the following are equivalent

(a) The Banach $*$-algebra $L^{1}(\mathbf{X})$ is commutative.

(b) The algebra $C_{c}^{\infty}(\mathbf{X})^{K}$ is commutative.

(c) The algebra $\mathbb{D}(\mathbf{X})$ is commutative.

Definition 4.2. $(G, K)$ is called a Gelfand pair if one, and hence all, of the conditions in Theorem 4.1 holds. In that case $\mathbf{X}$ is called a commutative space.

If $A$ is abelian, then $(A,\{e\})$ is a Gelfand pair. Similarly, if $K$ is a compact group that acts on the abelian group $A$ by group homomorphisms, i.e., $a \cdot(x y)=(a \cdot x)(a \cdot y)$ then $(G \rtimes K, K)$ is a Gelfand pair. One of the standard ways to decide if a given space is commutative is the following lemma:

Lemma 4.3. Assume there exists a continuous involution $\tau: G \rightarrow G$ such that $\tau(x) \in K x^{-1} K$ for all $x \in G$. Then $\mathbf{X}=G / K$ is commutative.

Proof. As $x \mapsto x^{-1}$ is an antihomomorphism it follows that $f \mapsto f^{\vee}$ is an antihomomorphism on $L^{1}(\mathbf{X})^{K}$. On the other hand if we define $f^{\tau}(x):=f(\tau(x))$ then $f \mapsto f^{\tau}$ is a homomorphism. But as $\tau(x)=k_{1} x^{-1} k_{2}$ it follows that $f^{\vee}=f^{\tau}$ for all $f \in L^{1}(\mathbf{X})^{K}$ and hence $L^{1}(\mathbf{X})^{K}$ is abelian.

Example 4.4. Let $G=\mathrm{SO}(d+1)$ and $K=\mathrm{SO}(d)$ is the group of rotations around the $e_{1}$-axis

$$
K=\left\{\left(\begin{array}{ll}
1 & 0 \\
0 & A
\end{array}\right) \mid A \in \mathrm{SO}(d)\right\}
$$

Then $K=\left\{k \in G \mid k\left(e_{1}\right)=e_{1}\right\}$. For $a \in G$ write $a=\left[a_{1}, \ldots, a_{d+1}\right]$ where $a_{j}$ are the row vectors in the matrix $a$. Then $a \cdot e_{1}=a_{1}$. If $x \in S^{d}$ set $a_{1}=x$ and extend $a_{1}$ to a positively oriented orthonormal basis $a_{1}, \ldots, a_{d+1}$ and set $a=\left[a_{1}, \ldots, a_{d+1}\right] \in G$. Then $a \cdot e_{1}=x$. This also shows that the stabilizer of $e_{1}$ is the group

$$
K=\left\{\left(\begin{array}{ll}
1 & 0 \\
0 & k
\end{array}\right) \mid k \in \mathrm{SO}(d)\right\} \simeq \mathrm{SO}(d) .
$$


Hence $S^{d}=G / K$. Let

$$
A:=\left\{a_{t}=\left(\begin{array}{ccc}
\cos (t) & -\sin (t) & 0 \\
\sin (t) & \cos (t) & 0 \\
0 & 0 & I_{d-1}
\end{array}\right) \mid t \in \mathbb{R}\right\} .
$$

Then every element $g \in G$ can be written as $k_{1} a k_{2}$ with $k_{1}, k_{2} \in K$ and $a \in A$. Define

$$
\tau(a)=\left(\begin{array}{cc}
-1 & 0 \\
0 & I_{d}
\end{array}\right) a\left(\begin{array}{cc}
-1 & 0 \\
0 & I_{d}
\end{array}\right)
$$

Then $\left.\tau\right|_{K}=$ id and $\tau(a)=a^{-1}$ if $a \in A$. Hence $\tau(x) \in K x^{-1} K$ which implies that $S^{d}$ is a commutative spaces.

Instead of working with the group it is better to work directly with the sphere. Think of $S^{d-1}$ as a subset of $S^{d}$ by $v \mapsto(0 v)$. If $u \in S^{d}$ then there is a $t$ and $v \in S^{d-1}$ such that

$$
u=\cos (t) e_{1}+\sin (t) v=k_{v} a_{t} e_{1}
$$

where $k_{v}$ is a rotation in $K$. The involution $\tau$ is now simply

$$
u \mapsto \cos (t) e_{1}-\sin (t) v=k_{v} a_{t}^{-1} e_{1}
$$

which can be rotated, using an element from $K$, back to $u$.

From now on $(G, K)$ will always-if nothings else is stated-denote a Gelfand pair and $\mathbf{X}$ will stand for a commutative space. We start with the simple Lemma, see [14], p. 75:

Lemma 4.5. Assume that $(G, K)$ is a Gelfand pair. Then $G$ is unimodular.

Recall that a function $\varphi: G \rightarrow \mathbb{C}$ is positive definite if $\varphi$ is continuous and for all $N \in \mathbb{N}$, all $c_{j} \in \mathbb{C}$, and all $x_{j} \in G, j=1, \ldots, N$, we have

$$
\sum_{i, j=1}^{N} c_{i} \overline{c_{j}} \varphi\left(x_{i}^{-1} x_{j}\right) \geq 0
$$

The following gives different characterizations of positive spherical functions. In particular, they arise as the $*$-homomorphisms of the commutative $B^{*}$-algebra $L^{1}(\mathbf{X})^{K}$ and as positive definite normalized eigenfunctions of $\mathbb{D}(\mathbf{X})$. Recall that we are always assuming that $G$ and hence also $\mathbf{X}$ is connected.

Theorem 4.6. Let $\varphi \in L^{\infty}(\mathbf{X})$. Then the following assertions are equivalent:

(a) $\varphi$ is $K$-bi-invariant and $L^{1}(\mathbf{X})^{K} \rightarrow \mathbb{C}, f \mapsto \widehat{f}(\varphi):=\int_{G} f(x) \overline{\varphi(x)} d \mu_{G}(x)$, is a homomorphism.

(b) $\varphi$ is continuous and for all $x, y \in G$ we have

$$
\int_{G} \varphi(x k y) d \mu_{K}(k)=\varphi(x) \varphi(y) .
$$

(c) $\varphi$ is $K$-bi-invariant, analytic, $\varphi(e)=1$ and there exists a homomorphism $\chi_{\varphi}: \mathbb{D}(\mathbf{X}) \rightarrow \mathbb{C}$ such that

$$
D \varphi=\chi_{\varphi}(D) \varphi
$$

for all $D \in \mathbb{D}(X)$.

The homomorphism in (a) is a $*$-homomorphism, if and only if $\varphi$ is positive definite.

Remark 4.7. We note that (7) implies that $\varphi$ is analytic because $\Delta \varphi=\chi_{\varphi}(\Delta) \varphi$ and $\Delta$ is elliptic.

Definition 4.8. $\varphi \in L^{\infty}(\mathbf{X})^{K}$ is called a spherical function if it satisfies the conditions in Theorem 4.6, 
Denote by $\mathcal{P}_{\mathrm{sp}}(\mathbf{X})$ the space of positive definite spherical functions. It is a locally compact Hausdorff topological vector space in the topology of uniform convergence on compact sets. The spherical Fourier transform $\mathcal{S}: L^{1}(\mathbf{X})^{K} \rightarrow \mathcal{C}\left(\mathcal{P}_{\mathrm{sp}}(\mathbf{X})\right)$ is the map

$$
\mathcal{S}(f)(\varphi)=\widehat{f}(\varphi):=\int_{G} f(x) \overline{\varphi(x)} d \mu_{G}(x)=\int_{G} f(x) \varphi\left(x^{-1}\right) d \mu_{G}(x) .
$$

The last equality follows from the fact that $\overline{\varphi(x)}=\varphi\left(x^{-1}\right)$ if $\varphi$ is positive definite. We note that $\widehat{f * g}=\widehat{f} \widehat{g}$.

Theorem 4.9. There exists a unique measure $\mu_{\mathcal{P}}$ on $\mathcal{P}_{\mathrm{sp}}(\mathbf{X})$ such that the following holds:

(a) If $f \in L^{1}(\mathbf{X})^{K} \cap L^{2}(\mathbf{X})$ then $\|f\|_{2}=\|\widehat{f}\|_{2}$.

(b) The spherical Fourier transform extends to a unitary isomorphism

$$
L^{2}(\mathbf{X})^{K} \rightarrow L^{2}\left(\mathcal{P}_{\mathrm{sp}}(\mathbf{X}), d \mu_{\mathcal{P}}\right)
$$

with inverse

$$
f(\cdot)=\int_{\mathcal{P}_{\mathrm{sp}}(\mathbf{X})} \widehat{f}(\varphi) \varphi(\cdot) d \mu_{\mathcal{P}}(\varphi)
$$

where the integral is understood in $L^{2}$-sense.

(c) If $f \in L^{1}(\mathbf{X})^{K} \cap L^{2}(\mathbf{X})$ and $\widehat{f} \in L^{1}\left(\mathcal{P}_{\mathrm{sp}}(\mathbf{X}), d \mu_{\mathcal{P}}\right)$ then (8) holds pointwise.

At this point we have not said much about the set $\mathcal{P}_{\mathrm{sp}}(\mathbf{X})$. However, it was proved in [46] that $\mathcal{P}_{\mathrm{sp}}(\mathbf{X})$ can always been identified with a subset of $\mathbb{C}^{s}$ for some $s \in \mathbb{N}$ in a very simple way.

Lemma 4.10. The algebra $\mathbb{D}(\mathbf{X})$ is finitely generated.

Proof. This is the Corollary on p. 269 in [37.

Let $D_{1}, \ldots, D_{s}$ be a set of generators and define a map

$$
\Phi: \mathcal{P}_{\mathrm{sp}}(\mathbf{X}) \rightarrow \mathbb{C}^{s}, \quad \varphi \mapsto\left(D_{1} \varphi(e), \ldots, D_{s} \varphi(e)\right)
$$

Let $\Lambda_{1}:=\Phi\left(\mathcal{P}_{\mathrm{sp}}(\mathbf{X})\right)$ with the topology induced from $\mathbb{C}^{s}, \Lambda:=\Phi\left(\operatorname{supp} \mu_{\mathcal{P}}\right)$ and $\widehat{\mu}:=\Phi^{*}\left(\mu_{\mathcal{P}}\right)$.

Theorem 4.11 (Ruffino, 46]). The map $\Phi: \mathcal{P}_{\mathrm{sp}}(\mathbf{X}) \rightarrow \Lambda$ is a topological isomorphism.

Remark 4.12. In 46 the statement is for the set of bounded spherical functions. But $\mathcal{P}_{\text {sp }}(\mathbf{X})$ is a closed subset of the set of bounded spherical functions, so the statement holds for $\mathcal{P}_{\mathrm{sp}}(\mathbf{X})$. Furthermore, we can choose the generators $D_{j}$ such that $\overline{D_{j}}=D_{j}$, ie., $D_{j}$ has real coefficients. If $\varphi \in \mathcal{P}_{\mathrm{sp}}(\mathbf{X})$, then $\bar{\varphi} \in \mathcal{P}$ sp $(\mathbf{X})$ and it follows that $\overline{\Lambda_{1}}=\Lambda_{1}$. We will always assume that this is the case.

For $\lambda \in \Lambda_{1}$ we let $\varphi_{\lambda}:=\Phi^{-1}(\lambda)$. We view the spherical Fourier transform of $f \in L^{1}(\mathbf{X})^{K} \cap L^{2}(\mathbf{X})^{K}$ as a function on $\Lambda$ given by $\widehat{f}(\lambda):=\widehat{f}\left(\varphi_{\lambda}\right)$.

\section{Spherical Functions and Representations}

To extend Theorem 4.9 to all of $L^{2}(\mathbf{X})$ one needs to connect the theory of spherical functions to representation theory. In this section $(G, K)$ will always denote a Gelfand pair. A unitary representation $(\pi, \mathcal{H})$ of $G$ is called spherical if the space of $K$-fixed vectors

$$
\mathcal{H}^{K}:=\{u \in \mathcal{H} \mid(\forall k \in K) \pi(k) u=u\}
$$

is nonzero. If $(G, K)$ is a Gelfand pair then $\operatorname{dim} \mathcal{H}^{K} \leq 1$ for all irreducible unitary representations of $G$.

Lemma 5.1. Let $f \in L^{1}(\mathbf{X})^{K}$. Then $\pi(f) \mathcal{H} \subseteq \mathcal{H}^{K}$. 
Proof. We have for $k \in K$ :

$$
\pi(k) \pi(f) v=\int_{G} f(x) \pi(k x) v d x=\int_{G} f\left(k^{-1} x\right) \pi(x) v d x=\int_{G} f(x) \pi(x) v d x=\pi(f) v .
$$

For the following statement, see for example Proposition 6.3.1 in [14].

Lemma 5.2. If $\mathcal{H}$ is an irreducible unitary representation of $G$ then $\operatorname{dim} \mathcal{H}^{K} \leq 1$.

Corollary 5.3. Let $(\pi, \mathcal{H})$ be a irreducible unitary representation of $G$ such that $\mathcal{H}^{K} \neq\{0\}$. Then there exists a $*$-homomorphism $\chi_{\pi}: L^{1}(\mathbf{X})^{K} \rightarrow \mathbb{C}$ such that

$$
\pi(f) u=\chi_{\pi}(f) u
$$

for all $u \in \mathcal{H}^{K}$.

Proof. Let $e_{\pi} \in \mathcal{H}^{K}$ be an unit vector. As $\operatorname{dim} \mathcal{H}^{K}=1$ it follows that $\mathcal{H}^{K}=\mathbb{C} e_{\pi}$. It follows from Lemma 1.5 that $\pi(f) e_{\pi}=\left(\pi(f) e_{\pi}, e_{\pi}\right) e_{\pi}$. The lemma follows now by defining $\chi_{\pi}(f):=\left(\pi(f) e_{\pi}, e_{\pi}\right)$.

Using the heat-kernel one can show that $\mathcal{H}^{K} \subset \mathcal{H}^{\omega}$ but the following is enough for us.

Theorem 5.4. $\mathcal{H}^{K} \subseteq \mathcal{H}^{\infty}$.

Proof. It is enough to show that $e_{\pi} \in \mathcal{H}^{\infty}$. For that let $f \in C_{c}^{\infty}(\mathbf{X})$ be so that $\left(\pi(f) e_{\pi}, e_{\pi}\right) \neq 0$. This is possible by Lemma 1.5, Let

$$
h(x)=\int_{K} f(k x) d \mu_{K}(k) .
$$

Then

$$
\chi_{\pi}(f)=\left(\pi(h) e_{\pi}, e_{\pi}\right)=\left(\pi(f) e_{\pi}, e_{\pi}\right) \neq 0 .
$$

Hence

$$
e_{\pi}=\frac{1}{\chi_{\pi}(f)} \pi(h) e_{\pi} \in \mathcal{H}^{K} \cap \mathcal{H}^{\infty} .
$$

Theorem 5.5. Let $(\pi, \mathcal{H})$ be an irreducible spherical representation of $G$ and $e_{\pi} \in \mathcal{H}^{K}$ a unit vector. Then the function

$$
\varphi_{\pi}(x):=\left(e_{\pi}, \pi_{\pi}(x) e_{\pi}\right)
$$

is a positive definite spherical function. If $\varphi$ is a positive definite spherical function on $G$, then there exists an irreducible unitary representation $(\pi, \mathcal{H})$ of $G$ such that $\operatorname{dim} \mathcal{H}^{K}=1$ and $\varphi=\varphi_{\pi}$.

Proof. Here are the main ideas of the proof. First we note that

$$
\int_{K} \pi(k y) e_{\pi} d \mu_{K}(k)=\left(\pi(y) e_{\pi}, e_{\pi}\right) e_{\pi} .
$$

Hence

$$
\begin{aligned}
\int_{K} \varphi_{\pi}(x k y) d \mu_{K}(k) & =\left(\pi\left(x^{-1}\right) e_{\pi}, \int_{K} \pi(k y) e_{\pi}\right) d \mu_{K}(k) \\
& =\left(\pi\left(x^{-1}\right) e_{\pi},\left(\pi(y) e_{\pi}, e_{\pi}\right) e_{\pi}\right) \\
& =\left(e_{\pi}, \pi(x) e_{\pi}\right)\left(e_{\pi}, \pi(y) e_{\pi}\right) \\
& =\varphi_{\pi}(x) \varphi_{\pi}(y) .
\end{aligned}
$$

Hence $\varphi_{\pi}$ is a spherical function. It is positive definite because

$$
\sum_{i, j=1}^{N} c_{i} \overline{c_{j}} \varphi_{\pi}\left(x_{i}^{-1} x_{j}\right)=\left\|\sum_{i=1}^{N} c_{i} \pi\left(x_{i}\right) e_{\pi}\right\|^{2} \geq 0 .
$$


Theorem 5.6. Let $\varphi: G \rightarrow \mathbb{C}$ be a positive definite function. Then $\varphi \in \mathcal{P}_{\mathrm{sp}}(\mathbf{X})$ if and only if there exists a irreducible spherical unitary representation $(\pi, \mathcal{H})$ and $e_{\pi} \in \mathcal{H}^{K},\left\|e_{\pi}\right\|=1$ such that

$$
\varphi(g)=\left(e_{\pi}, \pi(g) e_{\pi}\right)
$$

Proof. We have already seen one direction. The other direction follows by the classical Gelfand-NaimarkSegal construction. Assume that $\varphi$ is a positive definite function. Let $\mathcal{H}$ denote the space of functions generated by linear combinations of $\ell(x) \varphi, x \in G$. Define

$$
\left(\sum_{j=0}^{N} c_{j} \ell\left(x_{j}\right) \varphi, \sum_{j=0}^{N} d_{j} \ell\left(y_{j}\right) \varphi\right)_{0}:=\sum_{i, j} c_{i} \overline{d_{j}} \varphi\left(x_{i}^{-1} y_{j}\right)
$$

(By adding zeros we can always assume that the sum is taking over the same set of indices.) Then (, ) 0 is a positive semidefinite Hermitian form on $\mathcal{H}_{0}$. Let $\mathcal{N}:=\left\{\psi \in \mathcal{H}_{0} \mid\|\psi\|_{0}=0\right\}$. Then $\mathcal{N}$ is $G$-invariant under left-translations and $G$ acts on $\mathcal{H}_{0} / \mathcal{N}$ by left-translation. (, ) $)_{0}$ defines an inner product on $\mathcal{H} / \mathcal{N}$ by $(f+\mathcal{N}, g+\mathcal{N}):=(f, g)$. Let $\mathcal{H}$ be the completion of $\mathcal{H}_{0} / \mathcal{N}$ with respect with the metric given by $\left(\right.$, ). Then $\mathcal{H}$ is a Hilbert space and the left translation on $\mathcal{H}_{0}$ induces a unitary representation $\pi_{\varphi}$ on $\mathcal{H}$. If $e$ is the equivalence class of $\varphi \in \mathcal{H}$, then as $\varphi$ is $K$-invariant and $\|\varphi\|_{0}=1, e \in \mathcal{H}^{K} \backslash\{0\}$ and $\left(e, \pi_{\varphi}(x) e\right)=\varphi(x)$.

For $\lambda \in \Lambda_{1}$ and $\varphi=\varphi_{\lambda}$ we denote the corresponding representation by $\left(\pi_{\lambda}, \mathcal{H}_{\lambda}\right)$. We fix once and for all a unit vector $e_{\lambda} \in \mathcal{H}_{\lambda}^{K}$. Let $\operatorname{pr}_{\lambda}=\int_{K} \pi_{\lambda}(k) d k$. Then $\operatorname{pr}_{\lambda}$ is the orthogonal projection $\mathcal{H}_{\lambda} \rightarrow \mathcal{H}_{\lambda}^{K}$, $\operatorname{pr}_{\lambda}(u)=\left(u, e_{\lambda}\right) e_{\lambda}$. Let $f \in L^{1}(\mathbf{X})$. Then, as $f$ is right $K$-invariant, we get $\pi_{\lambda}(f)=\pi_{\lambda}(f) \circ \operatorname{pr}_{\lambda}$. It therefore make sense to define a vector valued Fourier transform by

$$
\tilde{f}(\lambda):=\pi_{\lambda}(f) e_{\lambda}
$$

see [4]. We note that if $f$ is $K$-invariant, then $\widehat{f}(\lambda)=\left(\widetilde{f}(\lambda), e_{\lambda}\right)$.

If $f \in L^{1}(\mathbf{X}) \cap L^{2}(\mathbf{X})$ then

$$
\widetilde{\ell(x) f}=\pi_{\lambda}(x) \widetilde{f}(\lambda) \quad \text { and } \quad \operatorname{Tr}\left(\pi_{\lambda}(f)\right)=\left(\pi_{\lambda}(f) e_{\lambda}, e_{\lambda}\right) .
$$

Let $g=\int f^{*} * f(k x) d \mu_{K}$. Then $g$ is $K$-binvariant and $\widetilde{g}(\lambda)=\left(\pi_{\lambda}\left(f^{*}\right) \pi_{\lambda}(f) e_{\pi}, e_{\pi}\right)=\|\widetilde{f}(\lambda)\|^{2}$ which is integrable on $\Lambda$. Finally

$$
\|f\|^{2}=f^{*} * f(e)=g(e)=\int_{\Lambda} \widehat{g}(\lambda) d \widehat{\mu}(\lambda)=\int_{\Lambda}\|\widetilde{f}(\lambda)\|^{2} d \widehat{\mu}(\lambda) .
$$

Finally, if $\lambda \mapsto\left(\widetilde{f}(\lambda), e_{\lambda}\right)$ is integrable, then by the same argument as above

$$
f(x)=\ell\left(x^{-1}\right) f(e)=\left(\pi_{\lambda}\left(x^{-1}\right) \widetilde{f}(\lambda), e_{\lambda}\right) d \widehat{\mu}(\lambda)=\int_{\Lambda}\left(\widehat{f}(\lambda), \pi_{\lambda}(x) e_{\lambda}\right) d \widehat{\mu}(\lambda) .
$$

Thus we have proved the following theorem:

Theorem 5.7. The vector valued Fourier transform defines a unitary G-isomorphism

$$
L^{2}(\mathbf{X}) \simeq \int^{\oplus}\left(\pi_{\lambda}, \mathcal{H}_{\lambda}\right) d \widehat{\mu} .
$$

If $f \in L^{2}(\mathbf{X})$ is so that $\lambda \mapsto\|\widehat{f}(\lambda)\|$ is integrable, then

$$
f(x)=\int_{\Lambda}\left(\widehat{f}(\lambda), \pi_{\lambda}(x) e_{\lambda}\right) d \widehat{\mu} .
$$




\section{The Space of Bandlimited Functions}

As before $(G, K)$ denotes a Gelfand pair with $G$ connected and $\mathbf{X}=G / K$ the corresponding commutative space. In this section we introduce the space of bandlimited functions and prove a sampling theorem for the spaces $L_{\Omega}^{2}(\mathbf{X})$ of $\Omega$-bandlimited functions on $\mathbf{X}$.

Definition 6.1. Suppose $\Omega \subset \Lambda$ be compact. We say that $f \in L^{2}(\mathbf{X})$ is $\Omega$-bandlimited if $\operatorname{supp} \tilde{f} \subseteq \Omega$. $f$ is bandlimited if there exists $\Omega \subseteq \Lambda$ compact such that $f$ is $\Omega$-bandlimited.

We denote by $L_{\Omega}^{2}(\mathbf{X})$ the space of $\Omega$-bandlimited functions. As $\Omega$ will be fixed, we just say that $f$ is bandlimited if $f \in L_{\Omega}^{2}(\mathbf{X})$. Let $\phi=\varphi_{\Omega}$ be such that $\widetilde{\varphi}(\lambda)=\mathbf{1}_{\Omega} e_{\lambda}$. As $\Omega$ is compact it follows that $\phi \in L_{\Omega}^{2}(\mathbf{X})$. However, $\mathbf{1}_{\Omega}$ is in general not integrable as $\lambda \mapsto \mathbf{1}_{\Omega}(\lambda) e_{\lambda}$ is not necessarily continuous.

Lemma 6.2. We have

$$
\phi_{\Omega}(x)=\int_{\Omega} \varphi_{\lambda}(x) d \widehat{\mu}(\lambda)
$$

is $K$-invariant and positive definite. In particular, $\phi_{\Omega}^{*}=\phi_{\Omega}$.

Proof. The function $\lambda \mapsto\left(\mathbf{1}_{\Omega}(\lambda) e_{\lambda}, \pi_{\lambda}(x) e_{\lambda}\right)_{\lambda}$ is bounded by $\left|\left(e_{\lambda}, \pi_{\lambda}(x) e_{\lambda}\right)_{\lambda}\right| \mathbf{1}_{\Omega}(\lambda) \leq \mathbf{1}_{\Omega}(\lambda)$ and hence integrable. Therefore Theorem 4.9 implies that

$$
\phi_{\Omega}(x)=\int_{\Omega}\left(e_{\lambda}, \pi_{\lambda}(x) e_{\lambda}\right)_{\lambda} d \widehat{\mu}(\lambda)=\int_{\Omega} \varphi_{\lambda}(x) d \widehat{\mu}(\lambda) .
$$

We have

$$
\sum_{i, j} c_{i} \overline{c_{j}} \phi\left(x_{i}^{-1} x_{j}\right)=\int_{\Omega} \sum_{i, j} c_{i} \overline{c_{j}} \varphi_{\lambda}\left(x_{i}^{-1} x_{j}\right) d \widehat{\mu}(\lambda) \geq 0
$$

as the spherical functions $\varphi_{\lambda}, \lambda \in \Omega$, are positive definite.

Theorem 6.3. $L_{\Omega}^{2}(\mathbf{X})$ is a reproducing kernel Hilbert space with reproducing kernel $K(x, y)=\phi_{\Omega}\left(y^{-1} x\right)$. Furthermore, the orthogonal projection $L^{2}(G) \rightarrow L_{\Omega}^{2}(\mathbf{X})$ is given by $f \mapsto f * \phi_{\Omega}$.

Proof. We have for $f \in L_{\Omega}^{1}(\mathbf{X})$

$$
\left|\int_{\Lambda}\left(\tilde{f}(\lambda), \pi_{\lambda}(x) e_{\lambda}\right)_{\lambda} d \widehat{\mu}(\lambda)\right| \leq \int_{\Omega}\|\widetilde{f}(\lambda)\|_{\lambda} d \widehat{\mu}(\lambda) \leq|\Omega|^{1 / 2}\|\widetilde{f}\|^{2}
$$

where $|\Omega|$ denotes the volume $\int_{\Omega} d \widehat{\mu}$ of $\Omega$ which is finite as $\Omega$ is compact. It follows that

$$
\begin{aligned}
f(x) & =\int_{\Omega}\left(\tilde{f}(\lambda), \pi_{\lambda}(x) e_{\lambda}\right)_{\lambda} d \widehat{\mu}(\lambda) \\
& =\int_{\Lambda}\left(\tilde{f}(\lambda), \pi_{\lambda}(x) \mathbf{1}_{\Omega}(\lambda) e_{\lambda}\right)_{\lambda} d \widehat{\mu}(\lambda) \\
& =\int_{\mathbf{X}} f(y) \overline{\ell(x) \phi_{\Omega}(y)} d y \\
& =\int_{\mathbf{X}} f(y) \phi_{\Omega}\left(y^{-1} x\right) d y \\
& =f * \varphi_{\Omega}(x) .
\end{aligned}
$$

Thus $L_{\Omega}^{2}(\mathbf{X})$ is a reproducing kernel Hilbert space with reproducing kernel $K(x, y)=\phi_{\Omega}\left(y^{-1} x\right)$. The rest follows now from Proposition 2.2 .

Let us point out the following consequence of Proposition 2.2

Corollary 6.4. Let $f \in L^{2}(G)$. Then $f \in L_{\Omega}^{2}(\mathbf{X})$ if and only if $f * \phi_{\Omega}=f$. 


\section{The Bernstein Inequality and sampling of Bandlimited Functions}

The definition of the topology on $\Lambda$ inspired by [46] ensures that the eigenvalues $c_{\lambda}$ for the Laplacian on $p_{\lambda}$ are bounded when $\lambda$ is in a compact set $\Omega$. This enables us to obtain

Lemma 7.1. For a compact set $\Omega \in \Lambda$ the functions in $L_{\Omega}^{2}$ are smooth and there is a constant $c(\Omega)$ such that the following Bernstein inequality holds

$$
\left\|\Delta^{k} f\right\|_{L^{2}} \leq c(\Omega)^{k}\|f\|_{L^{2}}
$$

Proof. As we have seen, each $f \in L_{\Omega}^{2}$ can be written

$$
f(x)=\int_{\Omega}\left(\widehat{f}(\lambda), \pi_{\lambda}(x) e_{\lambda}\right)_{\lambda} d \widehat{\mu}(\lambda)
$$

For fixed $\lambda$ the function

$$
t \mapsto\left(\tilde{f}(\lambda), \pi_{\lambda}\left(x e^{t X_{i}}\right) e_{\lambda}\right)_{\lambda}
$$

is differentiable as $e_{\lambda} \in \mathcal{H}_{\lambda}^{\infty}$. Thus, there exists a $t_{\lambda}$ between zero and $t$ such that

$$
\begin{aligned}
\frac{\left(\tilde{f}(\lambda), \pi_{\lambda}\left(x e^{t X_{i}}\right) e_{\lambda}\right)_{\lambda}-\left(\tilde{f}(\lambda), \pi_{\lambda}(x) e_{\lambda}\right)_{\lambda}}{t} & =\left(\tilde{f}(\lambda), \frac{\pi_{\lambda}\left(x e^{t X_{i}}\right) e_{\lambda}-\pi_{\lambda}(x) e_{\lambda}}{t}\right)_{\lambda} \\
& =\left(\tilde{f}(\lambda), \pi_{\lambda}\left(x e^{t_{\lambda} X_{i}}\right) \pi_{\lambda}\left(X_{i}\right) e_{\lambda}\right)_{\lambda} .
\end{aligned}
$$

Thus

$$
\begin{aligned}
\frac{f\left(x e^{t X_{i}}\right)-f(x)}{t} & =\int_{\Omega}\left(\tilde{f}(\lambda), \frac{\pi_{\lambda}\left(x e^{t X_{i}}\right) e_{\lambda}-\pi_{\lambda}(x) e_{\lambda}}{t}\right)_{\lambda} d \widehat{\mu}(\lambda) \\
& =\int_{\Omega}\left(\tilde{f}(\lambda), \pi_{\lambda}(x) \pi_{\lambda}\left(e^{t_{\lambda} X_{i}}\right) \pi_{\lambda}\left(X_{i}\right) e_{\lambda}\right)_{\lambda} d \widehat{\mu}(\lambda) \\
& \leq \int_{\Omega}\|\widetilde{f}\|_{\lambda}\left\|\pi_{\lambda}(x) \pi_{\lambda}\left(e^{t_{\lambda} X_{i}}\right) \pi_{\lambda}\left(X_{i}\right) e_{\lambda}\right\|_{\lambda} d \widehat{\mu}(\lambda) \\
& \leq \int_{\Omega}\|\widetilde{f}\|_{\lambda}\left\|\pi_{\lambda}\left(X_{i}\right) e_{\lambda}\right\|_{\lambda} d \widehat{\mu}(\lambda)
\end{aligned}
$$

Here we have used that $e_{\lambda}$ is a smooth vector for $\pi_{\lambda}$ and the unitarity of $\pi_{\lambda}$. Now

$$
\left\|\pi_{\lambda}\left(X_{i}\right) e_{\lambda}\right\|_{\lambda}^{2} \leq\left|\left(e_{\lambda}, \sum_{i} \pi_{\lambda}\left(X_{i}\right) \pi_{\lambda}\left(X_{i}\right) e_{\lambda}\right)_{\lambda}\right|=c_{\lambda}\left\|e_{\lambda}\right\|_{\lambda}^{2}
$$

Therefore the Lebesgue dominated convergence theorem ensures that

$$
\lim _{t \rightarrow 0} \frac{f\left(x e^{t X_{i}}\right)-f(x)}{t}=\int_{\Omega}\left(\tilde{f}(\lambda), \pi_{\lambda}(x) \pi_{\lambda}\left(X_{i}\right) e_{\lambda}\right)_{\lambda} d \widehat{\mu}(\lambda)
$$

which shows that $f$ is differentiable. Repeat the argument to show that $f$ is smooth and notice that

$$
\Delta^{k} f(x)=\int_{\Omega} c_{\lambda}^{k}\left(\tilde{f}(\lambda), \pi_{\lambda}(x) e_{\lambda}\right)_{\lambda} d \widehat{\mu}(\lambda)
$$

It then finally follows that

$$
\left\|\Delta^{k} f(x)\right\|_{L^{2}}^{2}=\int_{\Omega}\left|c_{\lambda}\right|^{2 k}\|\widetilde{f}\|_{w}^{2} d \mu(\lambda) \leq c(\Omega)^{2 k} \int_{\Omega}\|\widetilde{f}\|_{\lambda}^{2} d \widehat{\mu}(\lambda)
$$

We have thus proved the Bernstein inequality. 
Corollary 7.2. Let $\Omega \subseteq \Lambda$ be a compact set and define the neighborhoods $U_{\epsilon}$ by

$$
U_{\epsilon}=\left\{\exp \left(t_{1} X_{1}\right) \ldots \exp \left(t_{n} X_{n}\right) \mid\left(t_{1}, \ldots, t_{n}\right) \in[-\epsilon, \epsilon]^{n}\right\}
$$

It is possible to choose $\epsilon$ small enough that for any $U_{\epsilon}$-relatively separated family $x_{i}$ the functions $\ell\left(x_{i}\right) \phi$ form a frame for $L_{\Omega}^{2}$.

Corollary 7.3. Let $\Omega \subseteq \Lambda$ be a compact set and define the neighborhoods $U_{\epsilon}$ by

$$
U_{\epsilon}=\left\{\exp \left(t_{1} X_{1}\right) \ldots \exp \left(t_{n} X_{n}\right) \mid\left(t_{1}, \ldots, t_{n}\right) \in[-\epsilon, \epsilon]^{n}\right\}
$$

It is possible to choose $\epsilon$ small enough that for any $U_{\epsilon}$-relatively separated family $x_{i}$ and any partition of unity $0 \leq \psi_{i} \leq 1_{x_{i} U_{\epsilon}}$ the operator

$$
T_{1} f=\sum_{i} f\left(x_{i}\right) \psi_{i} * \phi
$$

is invertible on $L_{\Omega}^{2}$. If the functions $\ell\left(x_{i}\right) \phi$ also form a frame for $L_{\Omega}^{2}$, the functions $T_{1}^{-1}\left(\psi_{i} * \phi\right)$ provide a dual frame.

\section{Examples of Commutative Spaces}

In this section we give some examples of the theory developed in the previous section. We do not discuss the Riemannian symmetric spaces of the compact type as those can be found in [45].

8.1. The Space $\mathbb{R}^{d}$. The simplest example of a Gelfand pairs is $\left(\mathbb{R}^{d},\{0\}\right)$. The algebra of invariant differential operators is $\mathbb{D}\left(\mathbb{R}^{d}\right)=\mathbb{C}\left[\partial_{1}, \ldots, \partial_{d}\right]$ the polynomials in the partial derivatives $\partial_{j}=\partial / \partial x_{j}$. The positive definite spherical functions are the exponentials $\varphi_{\lambda}(x)=e^{i \lambda \cdot x}, \lambda \in \mathbb{R}^{d}$. Using $\partial_{1}, \ldots, \partial_{d}$ as generators for $\mathbb{D}\left(\mathbb{R}^{d}\right)$ Theorem 4.11 identifies $\Lambda$ with $i \mathbb{R}^{d}$ via the map $\varphi_{\lambda} \mapsto i\left(\lambda_{1}, \ldots, \lambda_{d}\right)$. Note the slight different from our previous notation for $\varphi_{\lambda}$.

We can also consider $\mathbb{R}^{d}$ as the commutative space corresponding to the connected Euclidean motion group $G=\mathrm{SO}(d) \ltimes \mathbb{R}^{d}$ with $K=\mathrm{SO}(d)$. The $K$-invariant functions are now the radial functions $f(x)=$ $F_{f}(\|x\|)$, where $F_{f}$ is a function of one variable. We have $\mathbb{D}\left(\mathbb{R}^{d}\right)=\mathbb{C}[-\Delta]$ and Theorem 4.11 now identifies the spectrum $\Lambda$ with $\mathbb{R}^{+}$. For $\lambda \in \mathbb{R}$ we denote by $\varphi_{\lambda}$ the spherical function with $-\Delta \varphi_{\lambda}=\lambda^{2} \varphi_{\lambda}$.

Denote by $J_{\nu}$ the Bessel-function

$$
J_{\nu}(r)=\frac{(r / 2)^{\nu}}{\Gamma(1 / 2) \Gamma(\nu+1 / 2)} \int_{-1}^{1} \cos (t r)\left(1-t^{2}\right)^{\nu-1 / 2} d t
$$

see [1], p. 144.

Lemma 8.1. $\varphi_{\lambda}(x)=\frac{2^{\frac{d-2}{2}} \Gamma\left(\frac{d}{2}\right)}{(\lambda\|x\|)^{\frac{d-2}{2}}} J_{(d-2) / 2}(\lambda\|x\|)=\frac{\Gamma\left(\frac{d}{2}\right)}{\sqrt{\pi} \Gamma\left(\frac{d-1}{2}\right)} \int_{-1}^{1} \cos (\lambda\|x\| t)\left(1-t^{2}\right)^{\frac{d-3}{2}} d t$.

Proof. Denote for the moment the right hand side by $\psi_{\lambda}$. Then $\psi_{\lambda}$ is analytic as cos is even. It is also an radial eigenfunction of $-\Delta$ with eigenvalue $\lambda^{2}$ and $\psi_{\lambda}(0)=1$. Now Theorem 4.6 implies that $\varphi_{\lambda}=\psi_{\lambda}$.

Remark 8.2. We note that we can write

$$
\varphi_{\lambda}(x)=\int_{S^{d-1}} e^{-i \lambda(\omega, x)} d \sigma(\omega)
$$

where $d \sigma$ is the normalized rotational invariant measure on the sphere.

It is easy to describe the representation $\left(\pi_{\lambda}, \mathcal{H}_{\lambda}\right)$ associated to $\varphi_{\lambda}$. For $\lambda \in \mathbb{R}^{*}$ set $\mathcal{H}_{\lambda}=L^{2}\left(S^{d-1}, d \sigma\right)=$ $L^{2}\left(S^{d-1}\right)$ and define

$$
\pi_{\lambda}((k, x)) u(\omega):=e^{-i \lambda(\omega, x)} u\left(k^{-1}(\omega)\right)
$$


We take the constant function $\omega \mapsto 1$ as normalized $K$-invariant vector $e_{\lambda}$. Then

$$
\left(e_{\lambda}, \pi_{\lambda}((k, x)) e_{\lambda}\right)=\int_{S^{d-1}} \overline{e^{-i \lambda(\omega, x)}} d \sigma(\omega)=\varphi_{\lambda}(x) .
$$

We refer to [4] for more information.

8.2. The Sphere $S^{d}$. Let $S^{d}=\left\{x \in \mathbb{R}^{d+1} \mid\|x\|=1\right\}$ be the unit sphere in $\mathbb{R}^{d+1}$. We refer to Chapter 9 of [16] and Chapter III in [51] for more detailed discussion on harmonic analysis and representation theory related to the sphere. In particular, most of the proofs can be found there. Recall from Example 4.4 that $S^{d}=G / K$ where $G=\mathrm{SO}(d+1)$ and $K=\mathrm{SO}(d)$ and that $S^{d}$ is a commutative space.

For $d=1$ we have $S^{1}=\mathbb{T}=\{z \in \mathbb{C}|| z \mid=1\}$ is an abelian group and the spherical functions are just the usual characters $z \mapsto z^{n}$ (or, if we view $\mathbb{T}=\mathbb{R} / 2 \pi \mathbb{Z}$, the functions $\theta \mapsto e^{i n \theta}$ ). We therefore assume that $d \geq 2$, but we would also like to point out another special case. $\mathrm{SO}(3) \simeq \mathrm{SU}(2)$ and $\mathrm{SO}_{o}(4)=\mathrm{SU}(2) \times \mathrm{SU}(2)$. The group $K=\mathrm{SU}(2)$ is embedded as the diagonal in $\mathrm{SU}(2) \times \mathrm{SU}(2)$. Then

$$
S^{3}=\mathrm{SO}(4) / \mathrm{SO}(3) \simeq \mathrm{SU}(2) \simeq\{z \in \mathbb{H}|| z \mid=1\}
$$

and the $K$-invariant functions on $S^{3}$ corresponds to the central functions on $\mathrm{SU}(2)$, i.e. $f\left(k u k^{-1}\right)=f(u)$. Hence $\Lambda$, the set of spherical representations, is just $\widehat{\mathrm{SU}(2)}$, the set of equivalence classes of irreducible representations of $\mathrm{SU}(2)$ and the spherical functions are

$$
\varphi_{\pi}=\frac{1}{d(\pi)} \operatorname{Tr} \pi=\frac{1}{d(\pi)} \chi_{\pi}
$$

where $d(\pi)$ denotes the dimension of $V_{\pi}$. We will come back to this example later.

Denote by $\mathfrak{g}=\mathfrak{s o}(d+1)=\left\{X \in M_{d+1}(\mathbb{R}) \mid X^{T}=-X\right\}$ the Lie algebra of $G$. We can take $\langle X, Y\rangle=$ $-\operatorname{Tr}(X Y)$ as a $K$-invariant inner product on $\mathfrak{g}$. Then

$$
\mathfrak{k}=\left\{\left(\begin{array}{cc}
0 & 0 \\
0 & Y
\end{array}\right) \mid Y \in \mathfrak{s o}(d)\right\} \simeq \mathfrak{s o}(d)
$$

and $\mathfrak{s}=\mathfrak{k}^{\perp}$ is given by

$$
\mathfrak{s}=\left\{X(v)=\left(\begin{array}{cc}
0 & -v^{T} \\
v & 0
\end{array}\right) \mid v \in \mathbb{R}^{d}\right\} \simeq \mathbb{R}^{d} .
$$

A simple matrix multiplication shows that $k X(v) k^{-1}=X(k(v))$ where we have identified $k \in \mathrm{SO}(d)$ with its image in $K$. It follows that the only invariant polynomials on $\mathfrak{s}$ are those of the form $p(X(v))=q\left(\|v\|^{2}\right)$ where $q$ is a polynomial of one variable. It follows that $\mathbb{D}\left(S^{d}\right)=\mathbb{C}[\Delta]$ where $\Delta$ now denotes the Laplace operator on $S^{d-1}$. Thus $\mathbb{D}\left(S^{d}\right)$ is abelian and hence $S^{d}=\mathrm{SO}(d+1) / \mathrm{SO}(d)$ is a commutative space.

Recall that a polynomial $p(x)$ on $\mathbb{R}^{d+1}$ is homogeneous of degree $n$ if $p(\lambda x)=\lambda^{n} p(x)$ for all $\lambda \in \mathbb{R} . p$ is harmonic if $\Delta_{\mathbb{R}^{d+1}} p=0$. Denote by $\mathcal{H}_{n}$ the space of harmonic polynomials that are homogeneous of degree $n$ and set

$$
\mathcal{Y}_{n}:=\left.\mathcal{H}_{n}\right|_{S^{d}}=\left\{\left.p\right|_{S^{d}} \mid p \in \mathcal{H}_{n}\right\}
$$

As the action of $G$ on $\mathbb{R}^{d+1}$ commutes with $\Delta_{\mathbb{R}^{d+1}}$ it follows that each of the spaces $\mathcal{Y}_{n}$ are $G$-invariant. Denote the corresponding representation by $\pi_{n}$. Thus $\pi_{n}(a) p(x)=p\left(a^{-1} x\right)$ for $p \in \mathcal{Y}_{n}$.

Theorem 8.3. The following holds:

(a) $\left(\pi_{n}, \mathcal{Y}_{n}\right)$ is an irreducible spherical representation of $\mathrm{SO}_{o}(d+1)$.

(b) If $(\pi, V)$ is an irreducible spherical representation of $G$ then there exists an $n$ such that $(\pi, V) \simeq$ $\left(\pi_{n}, \mathcal{Y}_{n}\right)$

(c) $\operatorname{dim} \mathcal{Y}_{n}=(2 n+d-1) \frac{(d+n-2) !}{(d-1) ! n !}=: d(n)$.

(d) $-\left.\Delta\right|_{\mathcal{Y}_{n}}=n(d+n-1)$. 
(e) $L^{2}\left(S^{d}\right) \simeq_{G} \bigoplus_{n=0}^{\infty} \mathcal{Y}_{n}$. In particular, every $f \in L^{2}\left(S^{d}\right)$ can be approximated by harmonic polynomials.

The last part of the above theorem implies that $\Lambda=\mathbb{N}=\{0,1, \ldots\}$. We use this natural parametrization of $\Lambda$ rather than the one given in Section 5 .

For $\Omega \in \mathbb{N}$ the Paley-Wiener space for $\Omega$ is

$$
L_{\Omega}^{2}\left(S^{d}\right)=\left\{\left.p\right|_{S^{d}} \mid p \text { is a harmonic polynomial of degree } \leq \Omega\right\} .
$$

It is noted that $\operatorname{dim} L_{\Omega}^{2}\left(S^{d}\right)<\infty$ which is also the case in the more general case of compact Gelfand pairs.

The group $\mathrm{SO}(d)$ acts transitively on spheres in $\mathbb{R}^{d}$. Hence every $v \in S^{d}$ is $K$-conjugate to a vector of the form $(\cos (\theta), \sin (\theta), 0, \ldots, 0)^{T}$ and a function $f$ is $K$-invariant if and only if there exists a function $F_{f}$ of one variable such that

$$
f(v)=F_{f}(\cos (\theta))=F_{f}\left(\left(v, e_{1}\right)\right)=F_{f}\left(v_{1}\right) .
$$

In particular, this holds for the spherical function $\varphi_{n}(x)$ corresponding to the representation $\pi_{n}$ as well as the reproducing kernel $\phi$ of the space $L_{\Omega}^{2}\left(S^{d}\right)$. In fact, for $d \geq 2$ the spherical functions are determined by the Jacobi polynomials, or normalized Gegenbauer polynomials in the following manner: $F_{\phi_{n}}(t)=\Phi_{n}(t)$ or $\varphi_{n}(x)=\Phi_{n}\left(\left(x, e_{1}\right)\right)=\Phi_{n}(\cos (\theta))$, where

$$
\begin{aligned}
\Phi_{n}(\cos (\theta)) & ={ }_{2} F_{1}\left(n+d-1,-n, \frac{d}{2} ; \sin ^{2}(\theta / 2)\right) \\
& ={ }_{2} F_{1}\left(n+d-1,-n, \frac{d}{2} ; \frac{1-\cos (\theta)}{2}\right) \\
& =\frac{n !(d-2) !}{(n+d-2) !} C_{n}^{(d-1) / 2}(\cos (\theta)) .
\end{aligned}
$$

As the polynomials $\varphi_{n}(t)$ are real valued we can write the spherical Fourier transform as

$$
\widehat{f}(n)=\int_{S^{d}} f(x) \varphi_{n}(x) d \sigma(x)=\frac{\Gamma\left(\frac{d+1}{2}\right)}{\sqrt{\pi} \Gamma\left(\frac{d}{2}\right)} \int_{-1}^{1} F_{f}(t) \Phi_{n}(t)\left(1-t^{2}\right)^{\frac{d}{2}-1} d t
$$

with inversion formula

$$
f(x)=\sum_{n=0}^{\infty} d(n) \widehat{f}(n) \varphi_{n}(x)=\sum_{n=0}^{\infty} d(n) \widehat{f}(n) \Phi_{n}\left(\left(x, e_{1}\right)\right) .
$$

In particular the sinc-type function is given by

$$
\phi_{\Omega}(x)=F_{\phi_{\Omega}}\left(\left(x, e_{1}\right)\right)=\sum_{n=0}^{\Omega} d(n) \Phi_{n}\left(\left(x, e_{1}\right)\right) .
$$

Note also that we can write the convolution kernel $\phi_{\Omega}\left(a^{-1} b\right), a, b \in \mathrm{SO}(d+1)$ as $F_{\phi_{\Omega}}((x, y))$ if $x=b e_{1}$ and $y=a e_{1}$.

For $d=1$ the sphere is the torus $\mathbf{T}=\{z \in \mathbb{C}|| z \mid=1\}$ and $\varphi_{n}(z)=z^{n}$. Hence

$$
\phi_{\Omega}\left(e^{i t}\right)=\sum_{n=-\Omega}^{\Omega} e^{n i t}=\frac{\sin ((\Omega+1 / 2) t)}{\sin (t / 2)}
$$

is the Dirichlet kernel $D_{\Omega}$. In the higher dimensional cases the kernel $\phi_{\Omega}$ behaves very similar to the Dirichlet kernel. Here are some of its properties:

Lemma 8.4. Let the notation be as above. Then the following holds:

(a) $\phi_{\Omega}\left(e_{1}\right)=\sum_{n=0}^{\Omega} d(n)=\operatorname{dim} L_{\Omega}^{2}\left(S^{d}\right) \nearrow \infty$ as $\Omega \rightarrow \infty$.

(b) $\int_{S^{d}} \phi_{\Omega}(x) d \sigma(x)=1$. 
(c) $\left\|\phi_{\Omega}\right\|_{2}^{2}=\sum_{n=0}^{\Omega} d(n) \rightarrow \infty$ as $\Omega \rightarrow \infty$.

(d) If $f \in L^{2}\left(S^{d}\right)$, then $f * \phi_{\Omega}=\int_{S^{d-1}} f(x) F_{\phi_{\Omega}}((\cdot, x)) d \sigma(x) \underset{\Omega \rightarrow \infty}{\longrightarrow} f$ in $L^{2}\left(S^{d}\right)$.

Let $N(\Omega)=\operatorname{dim} L_{\Omega}^{2}\left(S^{d}\right)=1+d(1)+\ldots+d(\Omega)$. Then every set of points $\left\{\omega_{j} \in S^{d} \mid j=1, \ldots, N(\Omega)\right\}$ such that the functions $F_{\phi_{\Omega}}\left(\left(\cdot, \omega_{j}\right)\right)$ are linearly independent will give us a basis (and hence a frame) for $L_{\Omega}^{2}\left(S^{d}\right)$. Further $N(\Omega)$ is the minimal number of points so that the sampling will determine a arbitrary function $f \in L_{\Omega}^{2}\left(S^{d}\right)$. If $n>N(\Omega)$, then the functions $\left\{F_{\phi_{\Omega}}\left(\left(\cdot, \omega_{j}\right)\right)\right\}_{j=1}^{n}$ will form a frame if and only if the set is generating.

Let us come back to the special case $S^{3} \simeq \mathrm{SU}(2)$. The set $\Lambda \simeq \widehat{\mathrm{SU}(2)}$ is isomorphic to $\mathbb{N}$ in such a way that $d(n)=d\left(\pi_{n}\right)=n+1$. Every element in $\mathrm{SU}(2)$ is conjugate to a matrix of the form

We have

$$
u(\theta)=\left(\begin{array}{cc}
e^{i \theta} & 0 \\
0 & e^{-i \theta}
\end{array}\right) .
$$

It follows that

$$
\varphi_{n}(u(\theta))=\frac{1}{n+1} \chi_{\pi_{n}}(u(\theta))=\frac{1}{n+1} \frac{\sin ((n+1) \theta)}{\sin (\theta)} .
$$

$$
\begin{aligned}
\phi_{\Omega}(u(\theta)) & =\frac{1}{\sin (\theta)} \sum_{n=1}^{\Omega+1} \sin (n \theta) \\
& =\frac{1}{2 i \sin (\theta)}\left(\sum_{n=1}^{\Omega+1}\left(e^{i \theta}\right)^{n}-\sum_{n=1}^{\Omega+1}\left(e^{-i \theta}\right)^{n}\right) \\
& =\frac{\sin ((\Omega+2) \theta / 2) \sin ((\Omega+1) \theta / 2)}{\sin (\theta) \sin (\theta / 2)} .
\end{aligned}
$$

8.3. Symmetric Spaces of the Compact Type. We will not discuss the general case of symmetric spaces $\mathbf{X}=G / K$ of the compact type to avoid introducing too much new notation, but the general case follows very much the same line as the special case of the sphere. Recall that that "symmetric space of the compact type" means that the group $G$ is compact and there exists an involution $\tau: G \rightarrow G$ such that with $G^{\tau}=\{u \in G \mid \tau(u)=u\}$ we have

$$
\left(G^{\tau}\right)_{o} \subseteq K \subseteq G^{\tau} .
$$

An example is the sphere $S^{d}$ where as in the last subsection $G=\mathrm{SO}_{o}(d+1)$ and the involution $\tau$ is given by

$$
u \mapsto\left(\begin{array}{cc}
-1 & 0 \\
0 & I_{d}
\end{array}\right) u\left(\begin{array}{cc}
-1 & 0 \\
0 & I_{d}
\end{array}\right)=\left(\begin{array}{cc}
u_{11} & -v^{t} \\
-v & k
\end{array}\right)
$$

as in Example 4.4. All of those spaces are commutative. The spectral set $\Lambda$ is well understood, see [39], Theorem 4.1, p. 535. In particular $\Lambda$ is discrete. Each representation $\left(\pi_{\lambda}, \mathcal{H}_{\lambda}\right)$ occur with multiplicity one in $L^{2}(\mathbf{X})$. Denote the image by $\mathcal{Y}_{\lambda}$. Then if $\Omega$ is given, there exists a finite set $\Lambda(\Omega) \subset \Lambda$ such that

$$
L_{\Omega}^{2}(\mathbf{X})=\bigoplus_{\lambda \in \Lambda(\Omega)} \mathcal{Y}_{\lambda}
$$

and $N(\Omega)=\operatorname{dim} L_{\Omega}^{2}(\mathbf{X})$ is finite. In particular, only finitely many points are needed to determine the elements in $L_{\Omega}^{2}(\mathbf{X})$.

The spherical functions are well understood. They are given by the generalized hypergeometric functions (and Jacobi polynomials) of Heckman and Opdam [35]. Again

$$
\phi_{\Omega}=\sum_{\lambda \in \Lambda(\Omega)} d\left(\pi_{\lambda}\right) \varphi_{\lambda} \in C^{\omega}(\mathbf{X})
$$


the Dirichlet kernel. Furthermore, Lemma 8.4 holds true in the general case.

8.4. Gelfand pairs for the Heisenberg group. For the details in the following discussion we refer to [50] and [6]. We let $\mathbb{H}_{n}=\mathbb{C}^{n} \times \mathbb{R}$ denote the $2 n+1$-dimensional Heisenberg group with group composition

$$
(z, t)\left(z^{\prime}, t^{\prime}\right)=\left(z+z^{\prime}, t+t^{\prime}+\frac{1}{2} \operatorname{Im}\left(\bar{z} z^{\prime}\right)\right)
$$

Denoting $z=x+i y$ the Heisenberg group is equipped with the left and right Haar measure $d x d y d t$ where $d x, d y, d t$ are Lebesgue meaures on $\mathbb{R}^{n}, \mathbb{R}^{n}$ and $\mathbb{R}$ respectively. The group $K=\mathrm{U}(n)$ acts on $\mathbb{H}_{n}$ by group homomorphism given by

$$
k \cdot(z, t)=(k z, t) .
$$

Let $G=K \ltimes \mathbb{H}_{n}$. It follows that $L^{p}(G / K) \simeq L^{p}\left(\mathbb{H}_{n}\right)$ and $L^{p}(G / K)^{K} \simeq L^{p}\left(\mathbb{H}_{n}\right)^{K}=L^{p}\left(\mathbb{H}_{n}\right)_{\text {rad }}$. It is known [5] that the algebra $L^{1}\left(\mathbb{H}_{n}\right)^{K}$ of integrable radial functions on $\mathbb{H}_{n}$ is commutative and thus $(G, K)$ is a Gelfand pair. This is also the case for several other subgroups of $U(n)$ as shown in [5].

8.4.1. Representation Theory for $G$. A collection of important representations for the Heisenberg group are the Bargman representations for $\lambda>0$ given by

$$
\pi_{\lambda}(z, t) f(w)=e^{i \lambda t-\lambda \operatorname{Im}(w \bar{z}) / 2-\lambda|z|^{2} / 4} f(w+z)
$$

is the Bargman representation of the Fock space $\mathcal{F}_{\lambda}$ of entire functions on $\mathbb{C}^{n}$ for which

$$
\|F\|_{\lambda}^{2}=\left(\frac{\lambda}{2 \pi}\right)^{n} \int_{\mathbb{C}^{n}}|F(z)|^{2} e^{-\lambda|z|^{2} / 2} d z<\infty .
$$

For $-\lambda<0$ define the representations

$$
\pi_{-\lambda}(z, t) f(w)=\pi_{\lambda}(\bar{z}, t) f(w)
$$

on the anti-holomorphic functions $\overline{\mathcal{F}_{\lambda}}$. These representation are irreducible and the left regular representation of $\mathbb{H}_{n}$ on $L^{2}\left(\mathbb{H}_{n}\right)$ decomposes as

$$
\int_{\mathbb{R}^{*}}^{\oplus}\left(\pi_{\lambda}, \mathcal{F}_{\lambda}\right)|\lambda|^{n} d \lambda
$$

We should note that there are more irreducible representations than the $\pi_{\lambda}$, but they are one-dimensional and do not show up in the Plancherel formula (they are of Plancherel measure 0).

Let us now turn to the regular representation of $G$ on $L^{2}\left(\mathbb{H}_{n}\right)$ given by

$$
\ell(k, z, t) f\left(z^{\prime}, t^{\prime}\right)=f\left(k^{-1}\left(z^{\prime}-z\right), t^{\prime}-t-\frac{1}{2} \operatorname{Im}\left(z^{\prime} \bar{z}\right)\right)
$$

Notice that $U(n)$ acts only on the $z$-variable, and for fixed $k \in U(n)$ the elements $G_{k}=\left\{(k z, t) \mid(z, t) \in \mathbb{H}_{n}\right\}$ is a group isomorphic to $\mathbb{H}_{n}$. Thus the left regular representation of $G_{k}$ on $L^{2}\left(G_{k}\right)$ can be decomposed using the Bargman representations. We get

$$
\left(\ell, L^{2}\left(G_{k}\right)\right)=\int_{\mathbb{R}^{*}}^{\oplus}\left(\pi_{\lambda}^{k}, \mathcal{F}_{\lambda}\right)|\lambda|^{n} d \lambda
$$

where $\pi_{\lambda}^{k}(z, t)=\pi_{\lambda}(k z, t)$. Note that with $\nu(k) f(w)=f\left(k^{-1} w\right)$ we have

$$
\pi_{\lambda}^{k}(z, t)=\nu(k) \pi_{\lambda}(z, t) \nu(k)^{-1} .
$$

Denote by $\pi_{\lambda, m}$ the representation $\nu$ restricted to the homogeneous polynomials of degree $m V_{\lambda, m}$. Then $\left(\nu, \mathcal{F}_{\lambda}\right)$ decomposes into

$$
\bigoplus_{m=0}^{\infty}\left(\pi_{\lambda, m}, V_{\lambda, m}\right)
$$


Note that $\operatorname{dim}\left(V_{\lambda, m}\right)=2 m+n, \operatorname{dim}\left(V_{\lambda, m}^{K}\right)=1$. Let $H_{\lambda, m}$ be the Hilbert space spanned by $\pi_{\lambda}(G) u_{\lambda}$ with $u_{\lambda}$ in $V_{\lambda, m}^{K}$. The representations of $G$ on $H_{\lambda, m}$ thus obtained are irreducible [50, and provide us with a decomposition of the left regular representation of $G$ on $L^{2}\left(\mathbb{H}_{n}\right)$ :

$$
\left(\ell, L^{2}\left(\mathbb{H}_{n}\right)\right)=\bigoplus_{m=0}^{\infty} \int_{\mathbb{R}^{*}}^{\oplus}\left(\pi_{\lambda, m}, H_{\lambda, m}\right)|\lambda|^{n} d \lambda .
$$

8.4.2. Spherical functions. The bounded $U(n)$-spherical functions in this case are

$$
\phi_{\lambda, m}(z, t)=\int_{U(n)}\left(\pi_{\lambda}^{k}(z, t) u_{\lambda}, u_{\lambda}\right)_{\mathcal{F}_{\lambda}} d k=e^{i \lambda t} L_{m}^{(n-1)}\left(|\lambda||z|^{2} / 2\right) e^{-|\lambda||z|^{2} / 4}
$$

for $\lambda \in \mathbb{R} \backslash\{0\}$ and $m=0,1,2, \cdots$. Here $L_{m}^{(n-1)}$ is the Laguerre polonomial of degree $m$ and order $n-1$

$$
L_{m}^{(n-1)}(x)=\left(\begin{array}{c}
m+n-1 \\
m
\end{array}\right)^{-1} \sum_{k=0}^{m}(-1)^{k}\left(\begin{array}{c}
m+n-1 \\
m-k
\end{array}\right) \frac{x^{k}}{k !}
$$

In this fashion the spectrum for $L^{1}\left(\mathbb{H}_{n}\right)^{K}$ can be identified with the Heisenberg fan [50]

$$
\mathfrak{F}_{n}=\left\{((2 m+n)|\lambda|, \lambda) \mid m \in \mathbb{N}_{0}, \lambda \neq 0\right\} \cup \mathbb{R}_{+}
$$

with Plancherel measure supported on $\Lambda=\left\{((2 m+n)|\lambda|, \lambda) \mid m \in \mathbb{N}_{0}, \lambda \neq 0\right\}$ and given explicitly as

$$
\int_{\Lambda} F(\phi) d \mu(\phi)=\int_{\mathbb{R}^{*}} \sum_{m \in \mathbb{N}_{0}}(2 m+n) F\left(\phi_{\lambda, m}\right)|\lambda|^{n} d \lambda .
$$

As shown by [7] and more generally in [46] the topologies on $\mathfrak{F}_{n}$ and $\Lambda$ is the topology inherited from $\mathbb{R}^{2}$.

8.4.3. Sampling and oversampling of band-limited functions. Let $L_{\Omega}^{2}\left(\mathbb{H}_{n}\right)$ be the space of functions in $L^{2}\left(\mathbb{H}_{n}\right)$ with Fourier transform supported in

$$
\Omega=((2 m+n)|\lambda|, \lambda)|m=0, \cdots, M ; 0<| \lambda \mid \leq R\} .
$$

In this case the sinc-type function is given by the integral

$$
\begin{aligned}
\phi(z, t)= & \sum_{m=1}^{M} \int_{0}^{R} e^{i \lambda t} L_{m}^{(n-1)}\left(|\lambda||z|^{2} / 2\right) e^{-|\lambda||z|^{2} / 4} d \lambda \\
& \quad+\int_{0}^{R} e^{-i \lambda t} L_{m}^{(n-1)}\left(|\lambda||z|^{2} / 2\right) e^{-|\lambda||z|^{2} / 4} d \lambda \\
= & \sum_{m=1}^{M} \int_{0}^{R} 2 \cos (\lambda t) L_{m}^{(n-1)}\left(|\lambda||z|^{2} / 2\right) e^{-|\lambda||z|^{2} / 4} d \lambda .
\end{aligned}
$$

Let $x_{i} U$ with $x_{i} \in G$ be a cover of the group $G=\mathbb{H}_{n} \ltimes K$, then $x_{i} K U K$ covers the Heisenberg group $\mathbb{H}_{n}$. Let $\psi_{i}$ be a bounded partition of unity, which could for example characteristic functions for disjoint sets $U_{i} \in x_{i} U$. The operator $T$ then has the form

$$
T f=\sum_{i} f\left(x_{i} K\right) \psi_{i} * \phi
$$

where $\phi$ is given above. Choosing $x_{i}$ close enough we can invert $T$ to obtain sampling results.

Another interesting application is related to oversampling. Let $\widehat{\phi}$ be a compactly supported Schwartz function on $\mathbb{R}^{2}$ such that its restriction to $\Omega$ is $1_{\Omega}$. Let us say the support is in $\Omega_{1}$. According to [2] there is a Schwartz function $\phi$ on $\mathbb{H}_{n}$ such that its Fourier transform is equal to $\widehat{\phi}$ restricted to $\Omega_{1}$. The function $\phi$ 
is therefore both integrable and band-limited. Let $\phi_{1}$ be the sinc function associated to $\Omega_{1}$, then choosing $x_{i}$ close enough (closer than for the operator $T$ to ensure that also $T_{1}$ is invertible) the operator

$$
T_{1} f=\sum_{i} f\left(x_{i} K\right) \psi_{i} * \phi_{1}
$$

becomes invertible on $L_{\Omega_{1}}^{2}\left(\mathbb{H}_{n}\right)$. Therefore

$$
f=\sum_{i} f\left(x_{i} K\right) T_{1}^{-1}\left(\psi_{i} * \phi_{1}\right)
$$

with convergence in $L_{\Omega_{1}}^{2}\left(\mathbb{H}_{n}\right)$. For $f \in L_{\Omega}^{2}\left(\mathbb{H}_{n}\right)$ we then also get, since $f=f * \phi$, that

$$
f=\sum_{i} f\left(x_{i} K\right) T_{1}^{-1}\left(\psi_{i} * \phi_{1}\right) * \phi
$$

and this time with convergence in $L_{\Omega}^{2}\left(\mathbb{H}_{n}\right)$.

Remark 8.5. This oversampling situation is not possible for symmetric spaces of non-compact type. The reason is that there are no integrable band-limited functions with Fourier transform constant on a set with limit point.

\section{REFERENCES}

[1] N. Aronszajn, , Theory of reproducing kernels, Trans. Amer. Math. Soc., 68, (1950), 337-404.

[2] F. Astengo, B. Di Blasio and F. Ricci, F., Gelfand pairs on the Heisenberg group and Schwartz functions, J. Funct. Anal., 256, (2009),1565-1587.

[3] J. Benedetto, Irregular sampling and frames. In: Ed. C. Chui, Wavelets - A Tutorial in Theory and Applications. Academic Press, 1991, 1-63.

[4] J. Benedetto and W. Heller, Irregular sampling and the theory of frames. Part I, Note Mathematica, X, Suppl. 1 (1990), $103-125$.

[5] C. Benson, J. Jenkins and G. Ratcliff, On Gel' fand pairs associated with solvable Lie groups, Trans. Amer. Math. Soc., 321, (1990), 85-116.

[6] _ Bounded $K$-spherical functions on Heisenberg groups, J. Funct. Anal., 105, 2, (1992), 409-443

[7] Spectra for Gelfand pairs associated with the Heisenberg group, Colloq. Math., 71, (1996),305-328

[8] J. G. Christensen. Sampling in reproducing kernel Banach spaces on Lie groups. ArXiv, 2011.

[9] J. G. Christensen and G. Ólafsson, Examples of coorbit spaces for dual pairs, Acta Appl. Math. 107 (2009), 2548.

[10] Coorbit spaces for dual pairs, Appl. Comp. Harmonic Anal. 31, (2011), 303-324.

[11] J. G. Christensen, A. Mayeli, G. Ólafsson, Sampling on commutative spaces. In preparation.

[12] O. Christensen, An introduction to frames and Riesz bases, Applied and Numerical Harmonic Analysis. Birkhäuser, 2003.

[13] J. Dieudonneé, Grundzüge der modernen Analysis, Band 5/6. Vieweg 1979.

[14] G. van Dijk, Introduction to Harmonic Analysis and Generalized Gelfand Pairs. Studies in Mathematics 36, de Gruyter, 2009.

[15] R. J. Duffin and A. C. Schaeffer, A Class of Nonharmonic Fourier Series, Transactions of the American Mathematical Society, 72, 2, 1952, 341-366, American Mathematical Society

[16] J. Faraut, Analysis on Lie Groups, An Introduction. Cambridge studies in advanced mathematics 110. Cambridge University Press, 2008.

$[17]$

[18] H. G. Feichtinger, Discretization of convolution and reconstruction of band-limited functions from irregular sampling. Progress in approximation theory, 333345, Academic Press, 1991.

[19] H. G. Feichtinger, Coherent frames and irregular sampling. In: Ed. J. S. Byrnes, Recent advances in Fourier analysis and its applications, 427440, NATO Adv. Sci. Inst. Ser. C Math. Phys. Sci., 315, Kluwer Acad. Publ., 1990.

[20] H. G. Feichtinger, K. H. Grc̈henig, A unified approach to atomic decompositions via integrable group representations. In: Function Spaces and Applications, Lund, 1986. Lecture Notes in Math., 1302, 5273. Springer, (1988)

$[21] \ldots$ Multidimensional irregular sampling of band-limited functions in $L^{p}$-spaces. Multivariate approximation theory, IV (Oberwolfach, 1989), 135142, Internat. Ser. Numer. Math., 90, Birkhäuser, 1989.

[22] Banach spaces related to integrable group representations and their atomic decompositions. I. J. Funct. Anal. 86, 307340 (1989) 
[23] Banach spaces related to integrable group representations and their atomic decompositions. II. Monatsh. Math. 108, (1989), 129148.

[24] , Iterative reconstruction of multivariate band-limited functions from irregular sampling values. SIAM $J$. $M a t h$. Anal. 23 (1992), 244261.

[25] _ Irregular sampling theorems and series expansions of band-limited functions. J. Math. Anal. Appl. 167 (1992), 530556.

[26] _ Theory and practice of irregular sampling. In: Ed. J. Benedetto and M. Wi. Fraxier, Wavelets: mathematics and applications, 305363, Stud. Adv. Math., CRC.

[27] H. G. Feichtinger and S. S. Pandey, Error estimates for irregular sampling of band-limited functions on a locally compact abelian group. J. Math. Anal. Appl. 279 (2003), 380397.

[28] H. Feichtinger and I. A. Pesenson, Recovery of band-limited functions on manifolds by an iterative algorithm. Wavelets, frames and operator theory, 137152, In: Ed. C. Heil, P. E. T. Jorgensen, D. R. Larson (Ed.) Wavelets, Frames and Operator Theory, Contemp. Math., 345, 137-152, Amer. Math. Soc., 2004,

[29] A reconstruction method for band-limited signals on the hyperbolic plane. Sampl. Theory Signal Image Process. 4 (2005), 107119.

[30] G. B. Folland, A Course in Abstract Harmonic Analysis, Studies in Advanced Mathematics, CRC Press, 1995.

[31] H. Führ, Abstract Harmonic Analysis of Continuous Wavelet Transforms Lecture Notes in Mathematics, 1863 SpringerVerlag, 2005.

[32] H. Führ and K. Grëhenig, Sampling theorems on locally compact groups from oscillation estimates, Math. Z. 255 (2007), 177194.

[33] D. Geller and I. Z. Pesenson, Band-limited localized Parseval frames and Besov spaces on compact homogeneous manifolds. J. Geom. Anal. 21 (2011), 334371.

[34] K. Gröchenig , Acceleration of the frame algorithm, IEEE Transactions on Signal Processing, 41, 1993, 3331-3340,12

[35] G. Heckman and H. Schlichtkrull, Harmonic Analysis and Special Functions on Symmetric Spaces. Perspectives in Mathematics 16, Academic Press 1994.

[36] C. Heil, A Basis Theory Primer: Expanded Edition, Applied and Numerical Harmonic Analysis. Birkhäuser/Springer, 2011.

[37] S. Helgason, Differential operators on homogeneous spaces. Acta Math. 102 (1959), 249-299.

[38] S. Helgason, Differential Geometry, Lie Groups, and Symmetric Spaces, Amer. Math. Soc., Providence, RI, 2001.

[39] - Groups and Geometric Analysis, Academic Press, 1984.

[40] J. Higgins, Five short stories about the cardinal series, Bull. Amer. Math. Soc., 12 (1985), $45-49$.

[41] N. N. Lebedev, Special Functions \& their Applications. Dover 1972.

[42] Li, S., The theory of multiresolution analysis frames and applications, Ph.D. dissertation, University of Maryland Baltimore County, United States, 1993

[43] Lim, J. K., Neumann series expansion of the inverse of a frame operator, Commun. Korean Math. Soc., 13, 1998, 4, $791-800,1225-1763$

[44] G. Ólafsson and H. Schlichtkrull, Representation theory, Radon transform and the heat equation on a Riemannian symmetric space. Group Representations, Ergodic Theory, and Mathematical Physics; A Tribute to George W. Mackey. In: Contemp. Math., 449 (2008), 315-344.

[45] I. Pesenson, A discrete Helgason-Fourier transform for Sobolev and Besov functions on noncompact symmetric spaces. Radon transforms, geometry, and wavelets. In G. Ólafsson, E. L. Grinberg, D. Larson, Palle E. T. Jorgensen, P. R. Massopust, E. T. Quinto, and B. Rubin, Radon Transforms, Geometry, and Wavelets, Contemp. Math., 464, 231247, Amer. Math. Soc., 2008.

[46] F. F. Ruffino, The Topology of the Spectrum for Gelfand Pairs on Lie Groups, Bullettino U.M.I. (8), 10-B (2007), 569-579.

[47] E. F. G. Thomas, An infinitesimal characterization of Gel'fand pairs. Contemp. Math. 26 (1984), $379-385$.

[48] C. E. Shannon, A mathematical theory of communication. Bell System Tech. J. 27, (1948), 379-423, 623-656.

[49] R. S. Strichartz, Analysis of the Laplacian on the complete Riemannian manifold, J. Funct. Anal., 52, 1983, 1, 48-79

[50] Strichartz, R. S., $L^{p}$ harmonic analysis and Radon transforms on the Heisenberg group, J. Funct. Anal., 96, (1991),350406.

[51] M. Takeuchi, Modern Spherical Runctions. Transactions of Mathematical Monographs 135 , AMS 1994.

[52] H. Triebel, Spaces of Besov-Hardy-Sobolev type on complete Riemannian manifolds. Ark. Mat., 24 299-337, 1986.

[53] Characterizations of Besov-Hardy-Sobolev spaces: A unified approach. J. Approx. Theory 52, (1988), 162203.

[54] J. A. Wolf, Harmonic Analysis on Commutative Spaces. Mathematical Surveys and Monographs, 142. American Mathematical Society, Providence, RI, 2007.

[55] W. Rudin, Functional analysis. International Series in Pure and Applied Mathematics. McGraw-Hill Inc., New York, second edition, 1991. 
[56] A. I. Zayed, Advances in Shannon's Sampling Theory, CRC, 1993.

[57] _ A prelude to sampling, wavelets, and tomography. In: Ed. J. J. Benedetto and A. I. Zayed, Sampling, Wavelets, and Tomography, 132, Appl. Numer. Harmon. Anal., Birkhäuser, 2004.

Department of mathematics, University of Maryland, College Park

E-mail address: jens@math.umd.edu

Department of Mathematics, Louisiana State University, Baton Rouge, LA 70803, USA

E-mail address: olafsson@math.1su.edu 